University of Tennessee Health Science Center

UTHSC Digital Commons

\title{
Joint Coordination Variability In Anterior Cruciate Ligament Reconstructed Subjects During Stair Ambulation Using a Vector Coding Technique
}

Bryan R. Simrak

University of Tennessee Health Science Center

Follow this and additional works at: https://dc.uthsc.edu/dissertations

Part of the Investigative Techniques Commons, Other Analytical, Diagnostic and Therapeutic

Techniques and Equipment Commons, and the Therapeutics Commons

\section{Recommended Citation}

Simrak, Bryan R. (http://orcid.org/0000-0001-8006-7296), "Joint Coordination Variability In Anterior Cruciate Ligament Reconstructed Subjects During Stair Ambulation Using a Vector Coding Technique" (2018). Theses and Dissertations (ETD). Paper 474. http://dx.doi.org/10.21007/etd.cghs.2018.0465. 


\title{
Joint Coordination Variability In Anterior Cruciate Ligament Reconstructed Subjects During Stair Ambulation Using a Vector Coding Technique
}

\begin{abstract}
Anterior cruciate ligament $(\mathrm{ACL})$ rupture is a common injury, with an estimated incidence of 120,000 to 200,000 per year in the United States. ACL reconstruction surgery is the standard treatment for this injury to restore knee joint stability and function. While surgical reconstruction has been shown to restore laxity of the knee, current literature lacks consensus on return to normal knee joint kinematics following surgery. Additionally, re-injury is a major risk for those who return to sports activity after reconstruction surgery. Dynamical systems methods for quantifying joint coordination variability have been explored as a method for detecting differences between ACL reconstructed (ACLR) subjects and healthy control subjects. Specifically, altered joint coordination variability has been linked to lower extremity instability, which may indicate re-injury risk.

The aim of this study was to assess joint coordination and joint coordination variability using a vector coding technique in ACLR subjects after recovery and return to normal activity. Our hypothesis was that joint coordination variability of ten selected intra-limb knee-knee and knee-hip couplings would be altered in the ACLR group compared to a group of healthy control subjects based on previous findings using similar methods.

Thirty subjects (15 ACLR and 15 normal) were analyzed using a motion capture camera system and force plates. Subjects were asked to ascend a staircase in a step-over-step manner at a self-selected pace, turn around on the elevated platform, then descend from the platform down the steps and return to the starting location. We employed a vector coding method using a custom Matlab script to measure coupling angle variability of knee-knee and hip-knee coupled motion during the stair activity. Individuals with ACLR were found to have differences in joint coordination variability (both increased and decreased) in 5 of the 10 joint couplings analyzed as compared with a healthy control group during the stair descent activity.

The majority of differences were found to be reductions in variability in the ACLR group as compared with controls. It is believed that there is an optimal amount of variability in any motor system that differentiates between the ability to adapt to environmental instability and the risk for injury. Reduced joint coordination variability indicates avoidance of a particular movement and results in the inability to adapt movement strategies in a dynamic environment. Decreased variability in ACLR subjects has also been linked to re-injury in at least one prospective study. These results combined with previous works provide insight into coordinative function after ACLR and may be useful in improving rehabilitation protocols following surgery as well as identifying those at risk of re-injury.
\end{abstract}

\section{Document Type}

Thesis

Degree Name

Master of Science (MS)

Program

Biomedical Engineering

Research Advisor

Brooke A. Sanford, Ph.D. 


\section{Keywords}

ACL reconstruction, anterior cruciate ligament, joint coordination, stairs, variability, vector coding

\section{Subject Categories}

Analytical, Diagnostic and Therapeutic Techniques and Equipment | Investigative Techniques | Medicine and Health Sciences | Other Analytical, Diagnostic and Therapeutic Techniques and Equipment |

Therapeutics 


\title{
Joint Coordination Variability in Anterior Cruciate Ligament Reconstructed Subjects During Stair Ambulation Using a Vector Coding Technique
}

\author{
A Thesis \\ Presented for \\ The Graduate Studies Council \\ The University of Tennessee \\ Health Science Center
}

\author{
In Partial Fulfillment \\ Of the Requirements for the Degree \\ Master of Science \\ In the Joint Graduate Program in Biomedical Engineering and Imaging \\ From The University of Tennessee \\ and \\ The University of Memphis
}

By

Bryan R. Simrak

December 2018 
Copyright (C) 2018 by Bryan R. Simrak. All rights reserved. 


\section{ACKNOWLEDGEMENTS}

I'd like to thank Brooke A. Sanford, PhD and John L. Williams, PhD for all the help they've given me toward completing this work, and especially Dr. Sanford for allowing me to join the program as her graduate assistant. Their expert knowledge has helped me immensely with my studies. Thank you to Richard A. Smith, PhD and William M. Mihalko, $\mathrm{PhD}$ for their guidance and teaching, and for serving on my committee. I would also like to thank everyone in Biomedical Engineering for their individual contributions, both direct and indirect.

A special thank you to my family. They have provided me with unwavering support without which I would not be here today.

And finally, thank you to my amazing fiancé, Jessica. Without her endless patience and encouragement I could not have completed this work. 


\begin{abstract}
Anterior cruciate ligament (ACL) rupture is a common injury, with an estimated incidence of 120,000 to 200,000 per year in the United States. ACL reconstruction surgery is the standard treatment for this injury to restore knee joint stability and function. While surgical reconstruction has been shown to restore laxity of the knee, current literature lacks consensus on return to normal knee joint kinematics following surgery. Additionally, re-injury is a major risk for those who return to sports activity after reconstruction surgery. Dynamical systems methods for quantifying joint coordination variability have been explored as a method for detecting differences between ACL reconstructed (ACLR) subjects and healthy control subjects. Specifically, altered joint coordination variability has been linked to lower extremity instability, which may indicate re-injury risk.
\end{abstract}

The aim of this study was to assess joint coordination and joint coordination variability using a vector coding technique in ACLR subjects after recovery and return to normal activity. Our hypothesis was that joint coordination variability of ten selected intra-limb knee-knee and knee-hip couplings would be altered in the ACLR group compared to a group of healthy control subjects based on previous findings using similar methods.

Thirty subjects (15 ACLR and 15 normal) were analyzed using a motion capture camera system and force plates. Subjects were asked to ascend a staircase in a step-overstep manner at a self-selected pace, turn around on the elevated platform, then descend from the platform down the steps and return to the starting location. We employed a vector coding method using a custom Matlab script to measure coupling angle variability of knee-knee and hip-knee coupled motion during the stair activity. Individuals with ACLR were found to have differences in joint coordination variability (both increased and decreased) in 5 of the 10 joint couplings analyzed as compared with a healthy control group during the stair descent activity.

The majority of differences were found to be reductions in variability in the ACLR group as compared with controls. It is believed that there is an optimal amount of variability in any motor system that differentiates between the ability to adapt to environmental instability and the risk for injury. Reduced joint coordination variability indicates avoidance of a particular movement and results in the inability to adapt movement strategies in a dynamic environment. Decreased variability in ACLR subjects has also been linked to re-injury in at least one prospective study. These results combined with previous works provide insight into coordinative function after ACLR and may be useful in improving rehabilitation protocols following surgery as well as identifying those at risk of re-injury. 


\section{TABLE OF CONTENTS}

CHAPTER 1. INTRODUCTION .....................................................................................1

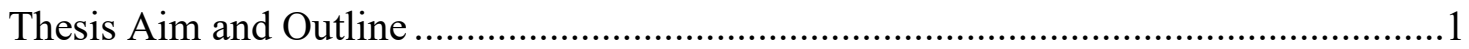

Role of the Anterior Cruciate Ligament ................................................................. 1

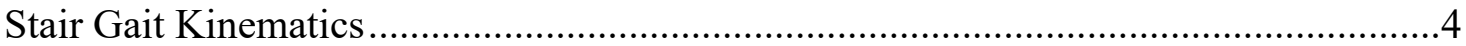

Lower Limb Biomechanics During Stair Ambulation ........................................

Altered Stair Kinematics with a Compromised ACL .............................................5

CHAPTER 2. JOINT COORDINATION VARIABILITY IN ANTERIOR CRUCIATE LIGAMENT RECONSTRUCTED SUBJECTS DURING STAIR DESCENT USING A VECTOR CODING TECHNIQUE .......................................10

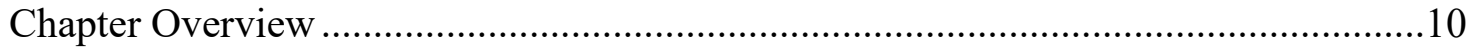

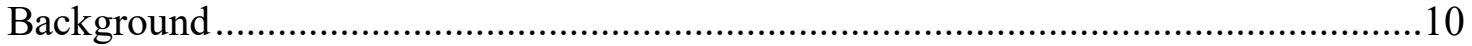

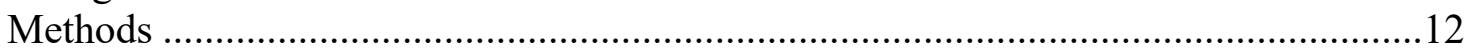

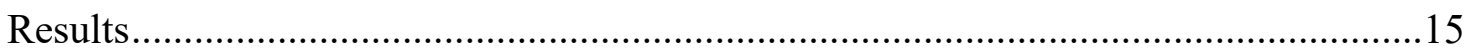

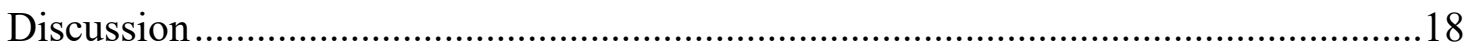

CHAPTER 3. ADDITIONAL RESULTS FOR OMITTED VARIABLES................21

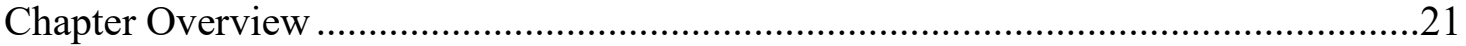

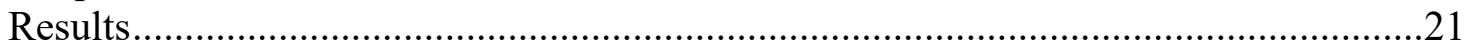

ACLR versus Healthy Control .............................................................................21

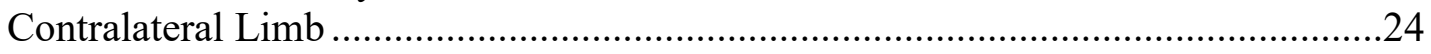

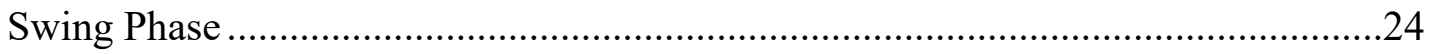

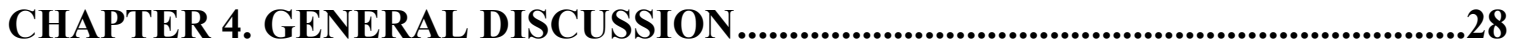

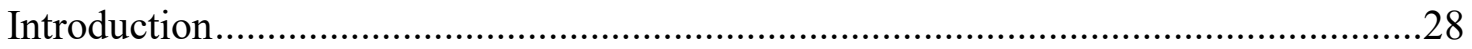

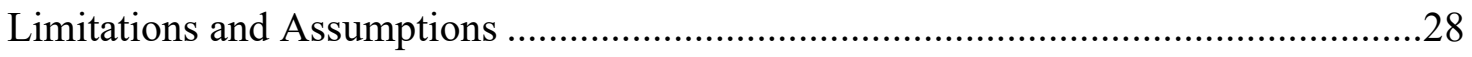

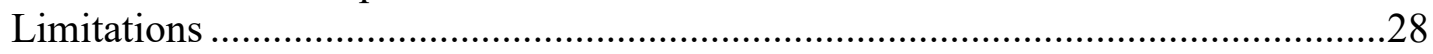

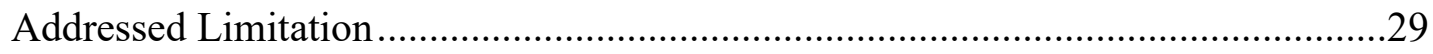

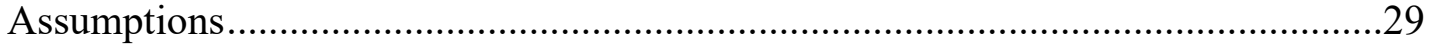

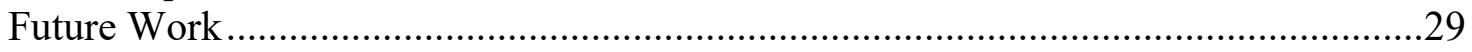

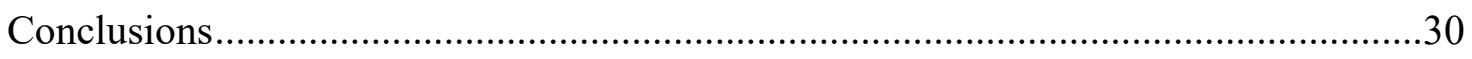

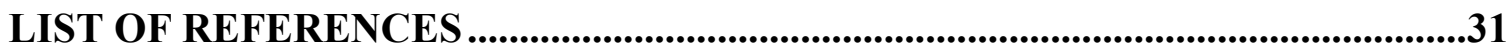

APPENDIX A. SAMPLE MATLAB SCRIPT USED TO CALCULATE THE

MEAN PHASE MAGNITUDE AND JOINT COORDINATION VARIABILITY ..36

APPENDIX B. COMPLETE SET OF JOINT COORDINATION

VARIABILITY ENSEMBLE AVERAGES FOR STAIR ASCENT AND

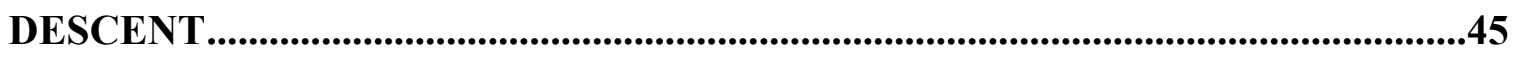

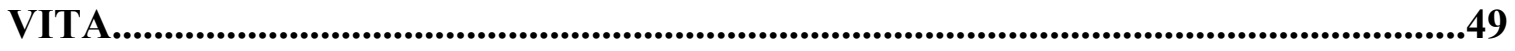




\section{LIST OF TABLES}

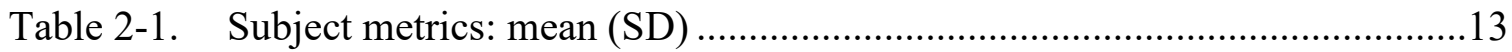

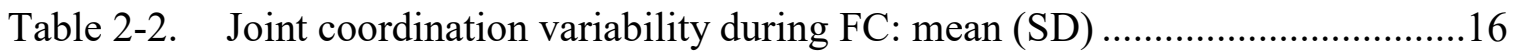

Table 2-3. Joint coordination variability during CL: mean (SD) ...............................16

Table 2-4. Coordination phase magnitude and classification during FC: mean (classification)

Table 2-5. Coordination phase magnitude and classification during CL: mean (classification)

Table 3-1. Reconstructed limb versus normal matched limb - joint coordination variability during stair ascent: mean $(\mathrm{SD})$

Table 3-2. Reconstructed limb versus normal matched limb - joint coordination variability during stair descent: mean $(\mathrm{SD})$

Table 3-3. Reconstructed limb versus contralateral limb - joint coordination variability during stair ascent: mean (SD)

Table 3-4. Contralateral limb versus normal matched contralateral limb - joint coordination variability during stair ascent: mean (SD)

Table 3-5. Reconstructed limb versus contralateral limb - joint coordination variability during stair descent: mean (SD).

Table B-1. Joint coordination variability during stair ascent: mean (SD) .45

Table B-2. Joint coordination variability during stair descent: mean (SD) 


\section{LIST OF FIGURES}

Figure 1-1 Origins and attachment points of the AM and PL bundles of the ACL within the intercondylar area of the knee joint .......................................... 3

Figure 1-2. The sub-phases of gait during stair ascent and stair descent .......................6

Figure 2-1. Locations of the retroreflective markers relative to the bony anatomy of

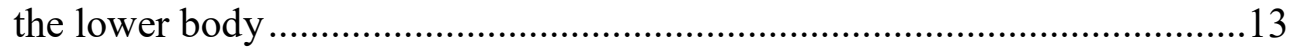




\section{CHAPTER 1. INTRODUCTION}

\section{Thesis Aim and Outline}

The goal of this study was to determine if there were differences in knee joint coordination variability for unilaterally anterior cruciate ligament (ACL) reconstructed persons as compared with subjects that had no history of lower extremity injury. People with deficient ACLs have been shown to have an increased risk of developing osteoarthritis that is not completely mitigated by reconstruction, [1] and reconstructed individuals have a high incidence rate of a second rupture [2]. The reasons for these statistics are not fully understood and may be linked to changes in lower body mechanics after surgery. We hypothesized that the ACL group would exhibit an altered standard deviation of the group average coupling angle resulting from instability in the affected joint. To explore this hypothesis, we analyzed data from a group of 30 subjects - $15 \mathrm{ACL}$ reconstructed and 15 healthy - collected in the University of Tennessee Health Science Center's gait laboratory. The data was comprised of ground reaction force (GRF) and motion captured kinematics measurements acquired using force plates and a retroreflective marker tracking camera system respectively.

Chapter 1 is an overview of the literature describing the function and anatomy of the native ACL, expected stair ambulation kinematics for a normal subject, and finally, movement patterns for ACL injured and reconstructed subjects identified during a stair climbing task. Chapter 2 shows an analysis of the stair descent data collected in our laboratory using an approach centered around five couplings. The coupling selection is based on their significance in previous work involving joint coordination analyses, which are also discussed. Chapter 3 details the limitations of the study and its approach to the clinical problem of increased injury risk for ACL reconstructed subjects. It also provides additional analyses on the stair ascent data and contralateral limb joint coordination variability to provide a more complete picture of the changes in the affected group. This chapter concludes with suggestions for future work on investigations into coupling angle variability.

\section{Role of the Anterior Cruciate Ligament}

The knee relies on the ACL primarily for the limitation of excessive translation and stabilization. An ACL injury is typically diagnosed using the Lachman test, in which the thigh is held steady while the shank is manipulated to test the laxity of the knee. This test and the similar Anterior Drawer test have been confirmed to have very high sensitivity by researchers analyzing large numbers of patient examinations $[3,4]$. When the ACL is confirmed to have been ruptured, reconstructive surgery using a graft may be used to restore the functionality of the ligament. In ACL reconstruction, the surgeon's aim is to replace the damaged ligament with a tendon either from the same patient, known as an autograft, or an allograft from a donor, ideally implanted with the same tension and attachment locations of the original. However, the intricacies of this 
procedure may preclude the reconstructed limb from being restored to its exact state prior to the injury.

In addition to the ACL providing tension at the knee, it is believed that the ligament's mechanoreceptors significantly contribute to balance and stability. A metastudy on ACL-injured and ACL reconstructed subjects compared with controls by Relph et al studied the effects on proprioception as measured by six studies. They ultimately concluded that injured and reconstructed were both found to have reduced proprioception in the knee, despite some concerns about the quality of the instruments used [5]. The most commonly performed assessment in the analyzed studies was the joint position sense test, where the assessor places the joint at specific angles, returns the joint to a resting position, then instructs the subject to return the limb to the same joint angle. In a study by Wang et al, investigators used an isokinetic dynamometer to determine if there was a link between balance and proprioception. Their results showed that there was a strong correlation between balance and proprioception, with the most significant results at $45^{\circ}$ of knee flexion [6]. At least one study has also demonstrated that proprioception and balance are significantly different depending on the type of reconstruction performed [7]. These findings indicate that an activity with a balancing or foot placement component can potentially require new movement strategies after a reconstruction, particularly in the case of the single-bundle graft, which is discussed in greater detail later.

The specific anatomy and attachment points of the ACL allow it to support the knee in conjunction with the other three ligaments of the joint. The lateral and medial collateral ligaments are predominantly responsible for resistance against varus and valgus forces respectively, while the crossed posterior and anterior cruciate ligaments resist posterior and anterior forces as well as tibial torque. The ACL resides between the tibia and femur at the knee joint. At the tibia, it attaches to the upper surface on the frontal, medial side of the intercondylar area. It travels through the intercondylar area and makes a half turn before attaching to the bottom surface of the femur posterolaterally [8]. This ligament consists of two bundles of connective tissue known as the anteromedial (AM) bundle and the thicker posterolateral (PL) bundle. As their names indicate, the AM bundle is inserted medially and anteriorly at the tibial insertion site while the PL bundle inserts slightly posterior and lateral to its correlate [9]. Figure 1-1 shows the origins and attachment points of the bundles relative to each other during varying levels of knee flexion. The amount of tension and contributed stability of each bundle is dependent on the amount of flexion in the knee due to their positioning within the joint.

Researchers have found methods to measure the involvement of each bundle and determine the flexion angles at which each is most responsible for performing the functions of the ACL. In a study on cadaveric human knees, Zantop et al used a robotic system to place loads on the knee to simulate anteriorly-directed, varus, and rotational forces [10]. The results of the study showed that resecting the AM bundle made a statistical difference in anterior translation of the tibia when the knee was at $60^{\circ}$ and $90^{\circ}$ flexion, whereas removing a portion of the PL bundle significantly increased translation 


\section{ACL bundles behaviour with knee flexion}
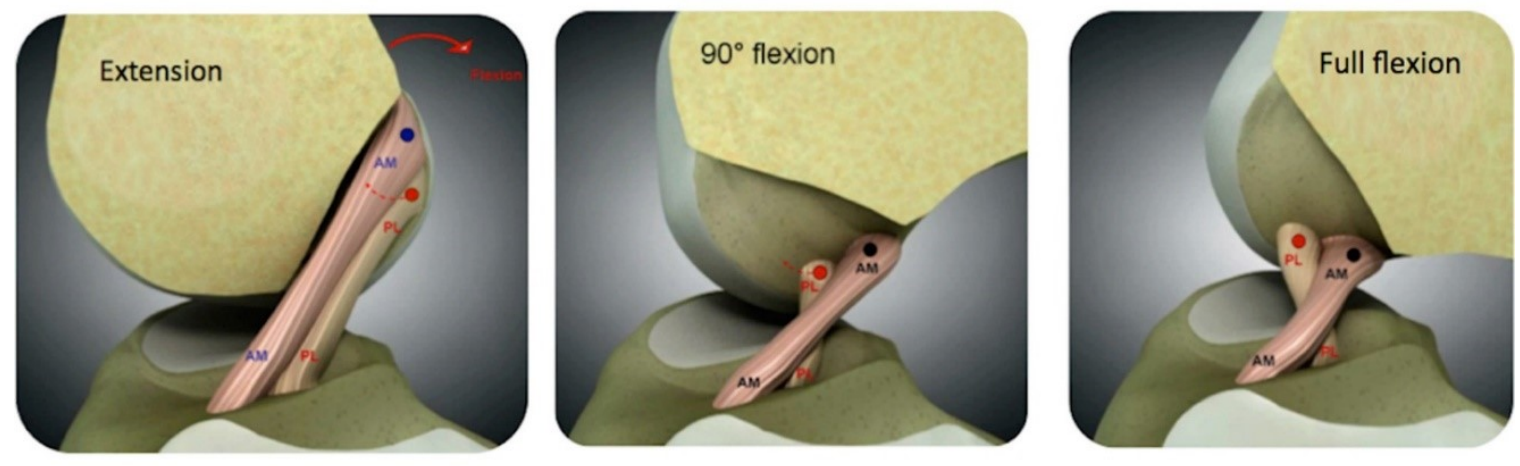

From extension to full flexion, the posterolateral bundle femoral insertion move in an arcuate path around the anteromedial bundle femoral insertion.

Anterior view of the knee ( $90^{\circ}$ of flexion) corresponding to arthroscopic vision

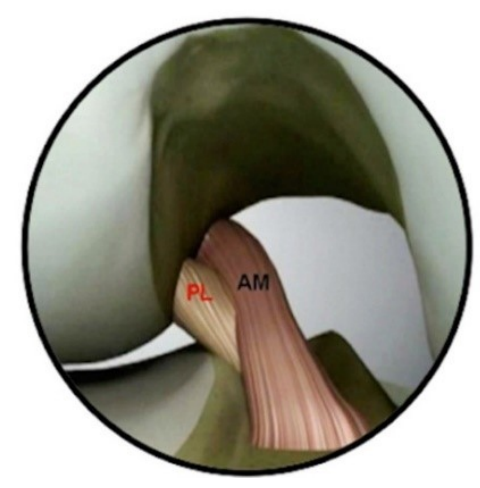

The PL bundle femoral insertion can be observed ahead and slightly below the AM bundle femoral insertion.

Figure 1-1 Origins and attachment points of the AM and PL bundles of the ACL within the intercondylar area of the knee joint

Reprinted with permission. Reproduced from Sonnery-Cottet, B., Colombet, P. Partial tears of the anterior cruciate ligament. Orthop Traumatol Surg Res, 2016; 102(1): p. S59-S67. Copyright (C) 2016 Elsevier Masson SAS. All rights reserved. 
when valgus and internal rotational forces were applied to the tibia at $0^{\circ}$ and $30^{\circ}$. These findings are in agreement with the majority of scientific articles and reviews on the role of bundles as studied in vitro $[8,9,11,12]$. Several studies have also proposed that a third fiber bundle, known as the intermediate bundle, is distinct from the other two, and that they have successfully separated it in cadaveric studies $[13,14]$. The complexity of this arrangement brings into question whether a graft using a single-bundled structure, such as the commonly used patellar tendon, will have any impact on its functionality. Additionally, we can infer from this information that observations made with the knee at certain degrees of flexion can be linked to a graft's ability to replicate the mechanics of a particular bundle.

Physicians can choose from a variety of graft types, and some purport greater analogy to the anatomy of the ACL. Patellar tendon, hamstring tendon, quadriceps tendon, cadaveric tissue, and double-bundle grafts each have advantages and disadvantages, and some may be more appropriate for specific patient situations due to variations in recovery time and pain. Some studies have shown statistical differences in laxity and failure among these grafts, marginally but significantly favoring the bonepatellar-tendon-bone (BPTB) over the hamstring tendon $[15,16]$. Studies on quadriceps tendon grafts are scarce but some have concluded it is at least equivalent to a hamstring graft [17]. Macaulay et al also reported that allograft versus autograft meta-studies found very minor differences between the two types and did not think that a conclusion could be made about whether one type was superior, though it is believed that an autograft will have better compatibility with the patient [15]. Double-bundled autografts are theoretically the most anatomically equivalent reconstruction of the ACL, and results for comparison analyses are generally in favor of the double-bundle albeit with relatively small differences in measurements $[18,19,20]$. Most notably, several of these studies have found that the double-bundle graft more closely restored the internal rotation of the knee to that of the native ACL and rupture rate was lower as compared with the single-bundle. Therefore, we have a need to evaluate the alterations in knee kinematics for single-bundle graft types in terms of whether they have an impact on movement mechanics.

\section{Stair Gait Kinematics}

\section{Lower Limb Biomechanics During Stair Ambulation}

Considerable effort has been made to fully characterize the kinematics and kinetics of stair gait using combinations of force plates, electromyographic measures, motion capture systems, electrogoniometers, and radiostereometric analysis. Since stairs are a well-practiced closed kinetic chain task found in everyday life that some people may traverse daily, stair climbing is a reliable way to measure lower body mobility and movement patterns that poses minimal risk of injury to subjects. For many people with lower extremity pathologies, being capable of negotiating stairs is required to maintain good quality of life. Additionally, stairs involve greater maximum flexion-extension 
range of the hip, knee, and ankle, and greater recruitment of the quadriceps as compared to walking [21].

McFadyen and Winter's measurements of stair kinematics and kinetics are often cited in modern work [22]. Their work was novel because it integrated three systems force plates, EMG, and a camera system - and accounted for intra-subject variability using a larger number of trials than previous similar studies. Their experiment also categorizes the various sub-phases in stair gait by the type of movement that is associated with each. In their work, stair ascent is divided into five categories: weight acceptance (starting with the beginning of stride at initial touch down), pull-up, and forward continuance for the stance phase, while the swing phase is separated into foot clearance and foot placement. Stair descent is separated similarly into weight acceptance, forward continuance, controlled lowering, leg pull-through, and foot placement, with the first three representing stance phase and the last two being swing phase. Zachazewski et al created a more detailed description of these sub-phases. In their article each of the subphases is described in terms of the subject's center of mass velocity and center of pressure in addition to the group averaged percent of stride, which has considerable intersubject variability [23]. These descriptions can be used to eliminate any ambiguity about the transition points between gait sub-phases. More recent studies on kinematics used more advanced motion tracking techniques to give a complete picture of the abductionadduction, flexion-extension (or dorsiflexion-plantarflexion), and rotation angles throughout stair stride for the hip, knee, and ankle simultaneously [21,24]. As expected, the results show that the flexion angle has the greatest range during these movements, while changes in the abduction-adduction angles are the smallest. Each stair task involves a sub-phase with forward momentum (forward continuance) and one in which the subject must change the height of their center of mass (controlled lowering or pull up). Figure 1-2 illustrates the approximate positions of the lower extremities during each of these sub-phases. Lowering or raising the body is associated with large changes in the hip and knee flexion angles, forward continuance shows relatively greater tibial rotation, and changes in dorsi/plantarflexion of the ankle can be seen throughout both movements. These studies describe stair gait events by average percent of stride across subject groups. From these studies we can conclude that the primary advantage of stairs over another task are large knee flexion angles which create tension in both ACL bundles rather than just the bundle associated with a mostly extended knee position. Additionally, foot placement, static stride length across all subjects (determined by the depth of the steps), and the requirement for the subject to move his bodyweight vertically during a single leg stance differentiate stair ambulation from most other tasks and may induce observable variations in movement techniques.

\section{Altered Stair Kinematics with a Compromised ACL}

A common approach to determining the effect of anatomical changes to a patient's mobility is to measure the kinematics of the affected extremity and major adjacent joints in the kinetic chain. Theoretically, by comparing these results to the 

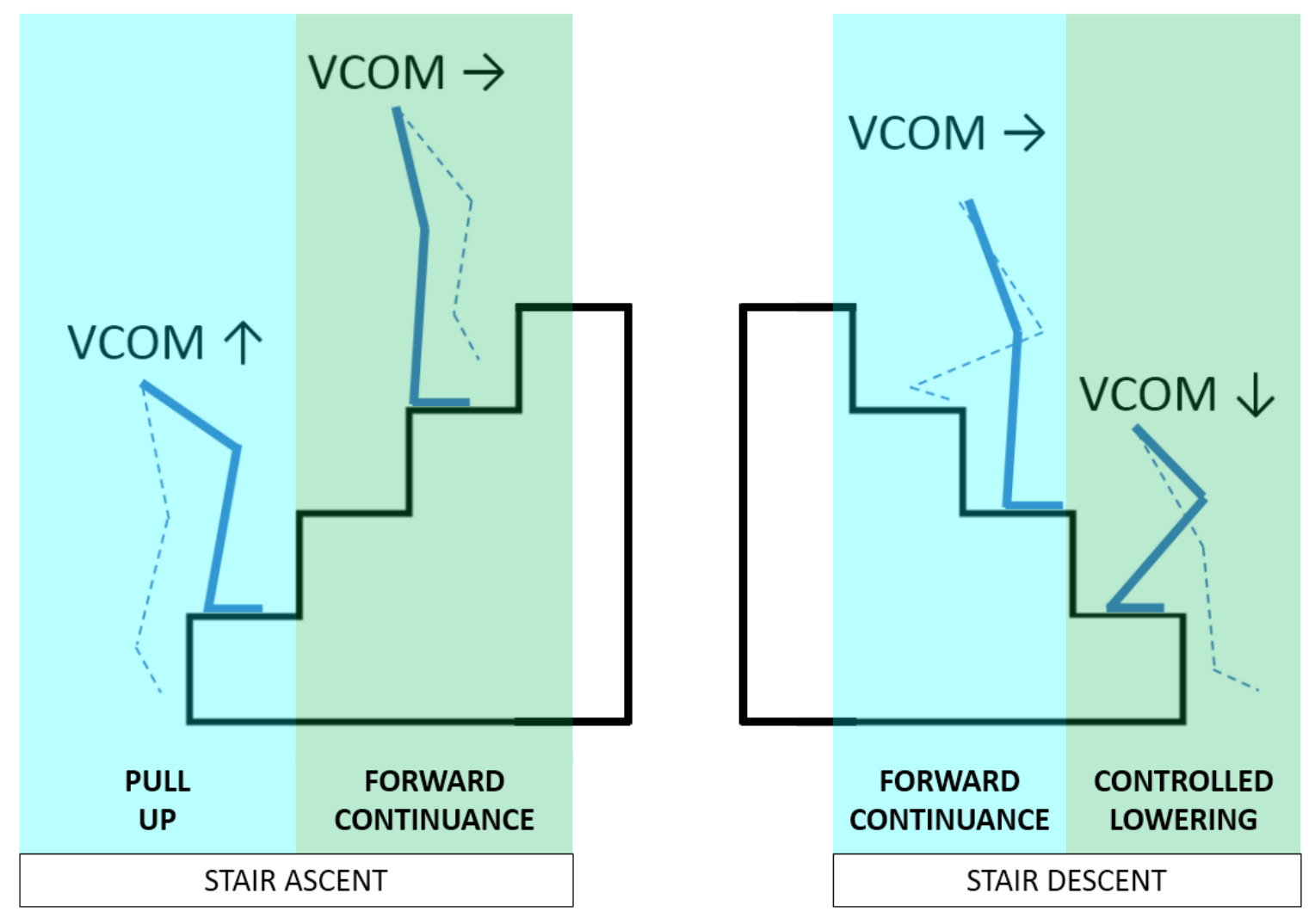

Figure 1-2. The sub-phases of gait during stair ascent and stair descent VCOM: velocity of center of mass 
expected kinematics of normal subjects we can understand what changes occur due to an ACL reconstruction and extrapolate the resultant effect on the subject. Since the ACL is primarily responsible for the limitation of excessive translation and rotation of the tibia, studies typically compare the maximum and range of angles required to achieve a task with those of a healthy control group. It is also very common for studies to break up the task into sub-phases, such as those previously discussed, or by percent of stride in order to refine the analysis.

The biomechanical differences identified using these methods for ACL deficient individuals are mixed. Several studies have analyzed this subject group during a stair climbing task, though some used the simpler step up or step down task in their experiments. Results largely reflect those of cadaveric studies on ruptured ACLs, though not all of those differences are found to be statistically significant in every study. This may be due to subjects adapting to the altered kinematics and existing differences could be attributed to avoidance of angles that are painful or unstable without the aid of an intact ACL. Lepley et al [25] compared controls with ACL-injured subjects before and after reconstruction and quantified the sagittal and frontal plane differences of the knee and hip. The results of their study were a bit different from others, indicating no kinematics differences during descent but significance in the knee flexion angle peak and range during ascent. They also observed larger hip abduction angle peaks and ranges when compared with controls. Another interesting finding was that all of the changes in kinematic variables remained significantly different from controls when the subjects were retested after their ACL surgery 6 months later. Unfortunately, Lepley did not look at the transverse plane, which has been identified as a point of interest in other studies. Berchuck et al [26] used a two-camera light-emitting diode tracking system to evaluate sagittal plane angles of the knee. Interestingly, their assessment showed no differences for either stair ascent or descent for kinematics of the knee. There was an observed difference in hip flexion angles during both tasks, but these differences did not result in a statistical significance for an alpha of 0.05 . Gao et al [27] performed the most comprehensive study on injured subjects during stair ambulation that was reviewed for this analysis. They investigated both deficient and reconstructed subjects during stair gait and found significant differences in all three anatomical planes for both groups as compared with uninjured controls, with the reconstructed differences generally being smaller. However, this investigation separated strides into 101 temporal intervals and does not appear to have made a statistical correction for multiple comparisons.

Researchers have also included a pivoting motion at the end of a stairs task to observe more rotation of the knee. Claes et al [28] used a camera system to capture threedimensional data while subjects descended a single step or descended and performed a pivot. Their analysis in the transverse plane reveals significantly increased internal rotation during descent and decreased tibial rotation for the pivot. Though the results of these studies are not in complete agreement with each other, they give an idea of what changes may persist after an ACL reconstruction and indicate what variables to focus on when analyzing those subjects.

Numerous studies have attempted to measure whether the ACL adequately restores the biomechanics of the knee after reconstruction surgery by observing subjects 
on stairs. Reconstructed subjects are typically either compared to a deficient group to measure similarities, contrasted against a normal control group, or juxtaposed with other graft types. Studies either compared the total angular range in a particular anatomical plane or the maximum or minimum angle at initial contact or during some other gait event. A single study involved prospective comparisons of subjects with ruptured ACLs, and concluded that kinematics variables they measured did not change significantly postsurgery. Brandsson et al [29] confirmed that knee laxity and joint angles of injured and reconstructed subjects. Their investigation involved a step-up task and did not find statistical differences between the injured and reconstructed groups, though they reported diminished laxity in the latter. Studies comparing to normal subjects show less agreement among their results than those of deficient subjects. Four of the reviewed studies observed differences in knee flexion angles, however none were in complete agreement. Gao et al found less extension in both ascent and descent, Lepley et al's measurements were not significant in descent but were in ascent, Hall et al observed differences in descent and not in ascent, and finally, Sole et al did not find any sagittal motion differences (though it should be noted that the subjects in the latter study were 20 years post-surgery) $[25,27,30,31]$. Three studies opted to observe transverse plane kinematics, though they did not all use directly comparable methods. Ristanis et al [32] used the descent and pivot task to detect increased knee rotation range, though they did so using a transitive comparison with the contralateral limb of the affected subjects rather than a direct one with the controls. The researchers did not give a reason as to why they did not make a direct comparison. As previously mentioned, Sole's methods uncovered no differences for either the ascent or descent task [31]. However, Gao et al [27] observed that reconstructed knees were more externally rotated in both tasks, albeit at discrete points in stride. Three studies calculated kinematics in the frontal plane with similar results to those of the frontal plane. Lepley and Sole did not find differences for either task, whereas Gao noted that knees were significantly more varus in mid-stance during both [25,27,31]. Lepley et al [25] were the only group to compare hip kinematics for reconstructed and healthy groups. As with their other findings, peak and range of angles were found to be the same in both frontal and sagittal planes during descent but were different during ascent. The lack of agreement among the results and methods in these studies indicates that there may be a need for the use of more complex methods of measurement rather than the traditional kinematics approach.

Previous works have shown that dynamical systems methods can be used to identify aberrant movement patterns [33,34]. These methods are useful for complex biological systems where there are multiple joints or segments contributing toward an end goal, such as completion of the stair climbing task where the segments of the lower body must coordinate to traverse the steps. Rather than looking only at one specific segment or joint, we can observe their motion relative to another contributor within the system [35]. Spinelli et al [33] describe several techniques that can be used to describe the coordinated movement within a system and present three examples where focusing solely on joint angles individually was not adequate to detect movement differences. Furthermore, van Emmerick and van Wegen have detailed the usefulness of comparing the variability of relative movement measurements. Their article explores the role of movement variability in biological systems and concludes that there is an optimal amount of variability that 
maximizes stability and efficiency in most cases, [36] a sentiment echoed in Stergiou et al's analysis of variability as an inherent component of human locomotion [37]. Hamill et al performed a study where they concluded that lower coordination variability also results in less distributed stress on the soft tissue, which may cause degenerative effects [38]. It has also been previously shown that relative movement variability measurements can successfully identify abnormal movement patterns not apparent in classical kinematics measurements [34]. With these discussions and the results of previous stair climbing studies in mind, we decided that an analysis using the vector coding method of quantifying joint coordination would be a novel approach to studying stair gait. 


\section{CHAPTER 2. JOINT COORDINATION VARIABILITY IN ANTERIOR CRUCIATE LIGAMENT RECONSTRUCTED SUBJECTS DURING STAIR DESCENT USING A VECTOR CODING TECHNIQUE}

\section{Chapter Overview}

Anterior cruciate ligament (ACL) reconstruction is a treatment for ACL ruptures used to restore function of the knee. The stair climbing activity is easy to perform in a laboratory setting and involves greater maximum flexion angles than walking. No study prior to this has evaluated joint coordination variability during a stair activity. Our study's aim was to assess joint coordination and joint coordination variability using a vector coding technique in ACLR subjects after recovery and return to normal activity. Data for ten selected intra-limb knee-knee and knee-hip couplings over two sub-phases of stair descent were collected for 15 ACLR subjects and 15 healthy control subjects. Joint coordination variability differences were found in 5 of the 10 couplings analyzed. Altered joint coordination variability has been shown to be an indicator of instability during a lower extremity ambulatory task. Reduced joint coordination variability indicates inability to adapt movement strategies and has been linked to re-injury.

\section{Background}

Anterior cruciate ligament (ACL) injury is a common knee disorder for participants in sports activities, particularly for high school-aged athletes [39]. Estimates for the number of ACL injuries in the United States are between 120,000 and 200,000 per year [40.41]. While reconstruction surgery can restore much of the lost function of the ligament to the knee, studies show that the risk for ACL reinjury may be as high as 25 times greater for those who return to athletics following recovery [42]. This presents a need for identifying mechanisms that can lead to repeat injury.

The kinematics of ACLR subjects negotiating stairs have been analyzed in depth by many research groups, however, some results are conflicting. A recent review of studies on the stair ambulation kinematics and kinetics of ACL reconstructed knees concluded that few studies found differences when compared with control subjects [43]. Several studies in the review found that the peak knee flexion at initial contact during stair ascent and descent was lower for reconstructed subjects, and a single study found lower peak knee varus, internal, and external rotation during descent, while most did not have any significant findings or only found differences in joint moments [43]. Cadaveric studies show that ACL injuries can result in changes in the knee joint such as excessive anterior translation and altered internal and external rotation of the tibia [8,44]. It has been shown that ACL reconstruction surgery is effective at restoring the ability of the knee joint to restrict anterior tibial translation, but several researchers have reported that tibial rotation continues to match that of ACL injured subjects during basic lower extremity tasks such as walking and single stair step-up [29,45]. Due to these inconsistent 
results, it is possible that focusing solely on kinematics might not paint a complete picture of changes in the knee joint after reconstruction surgery.

A dynamical systems approach focusing on joint coordination variability has been shown to highlight altered movement patterns in instances where there were no findings in traditional kinematic measurement comparisons [34,38]. In addition to allowing the observer to look at the relationship between two joints, joint coordination variability provides data throughout an entire movement, rather than requiring that the observer focus on extrema or a discrete time point. Furthermore, several research groups have extrapolated on the meaning of altered variability in movement tasks. It is theorized that there is an "optimal variability" in movement, where excess variability indicates instability while decreased variability suggests reduced adaptability, creating a greater risk of injury from unforeseen external variables such as those commonly seen in sports $[36,37]$.

Prior research with ACLR patients has attempted to quantify the differences in joint coordination variability compared to normal, healthy subjects during a variety of lower extremity tasks. Davis et al [46] used the vector coding technique to identify increased variability in ACLR subjects during walking for several hip-knee couplings. Gribbin et al [47] found decreased variability in some coupling and plane combinations and increased variability in others while ACLR subjects completed walking and jogging tasks. Pollard et al [48] observed their subjects during a more physically demanding cutting task and chose to also measure intra-joint coordination variability. These researchers found that there were differences in variability for the knee-flexion $/ \mathrm{knee-}$ abduction and knee-IE rotation/knee-abduction couplings, but nothing was found for knee-flexion/knee-IE rotation. Paterno et al [2] were able to prospectively identify ACLR subjects who would go on to incur a second ACL injury by analyzing joint coordination variability during a balancing task. They theorized that, due to changes in proprioception in the knee resulting from surgery, the subjects would take on new movement strategies involving the proximal and distal joints in the kinetic chain. Based on these studies, we attempted to find differences in joint coordination variability during stair descent, which is a common task that can be easily repeated in a clinical or physical therapeutic setting.

To date, only one study has used a dynamical systems method to compare ACL reconstructed subjects with control subjects during stair ambulation. Hsu et al [49] used the continuous relative phase and root-mean-square methods to measure differences in joint coordination for the hip-knee couplings in the sagittal plane during both stair ascent and stair descent. However, they did not compare the stride-to-stride variability nor did they include coordination in the coronal and transverse planes, which, may be of interest due to previous works that found surgery did not completely restore normal knee rotation and ab/adduction $[29,43,45]$. Therefore, the purpose of our study was to compare the intra- and inter-joint coordination variabilities of reconstructed and healthy control subjects during stair descent. We elected to focus on the descent portion of stair ambulation due to a higher prevalence of findings as compared to ascent in previous work with reconstructed subjects' kinetics and kinematics on stairs [43]. We hypothesized that the hip-knee and knee-knee joint coupling variabilities of the reconstructed subjects 
would be different from those of healthy controls due to alternate coordination techniques.

\section{Methods}

Stair ambulation data were collected for thirty subjects. Fifteen (nine male, six female) of these subjects had undergone unilateral ACL reconstruction surgery and had subsequently been cleared to return to physical activity. Eleven of these surgeries used a bone-patellar-tendon-bone graft, one used a hamstring tendon, two were cadaveric, and one was unknown. There was an average of 8.1 years ( $\mathrm{SD}=4.6$ years) between surgery and the date of the testing protocol ranging from 0.7 to 14.7 years. These subjects were compared with a control group of fifteen subjects with normal, healthy lower extremities and no recent injuries that could impair or alter movement of the lower body. Subjects that closely matched the BMI, age, and gender distribution of the ACLR subjects were selected for the analysis. Their metrics are summarized in Table 2-1. Subjects with a BMI of 35 or greater were excluded from the investigation due to potential inaccuracies resulting from soft tissue movement. Normal subjects were paired with ACLR subjects of similar metrics for the purpose of selecting the normal subjects' limbs to be analyzed.

The data were collected using a nine-camera motion capture system (Qualisys AB, Gothenburg, Sweden) at the University of Tennessee Health Science Center's gait laboratory. Subjects wore form-fitting clothing and retroreflective markers were attached to specific locations of the body including: anterior-superior iliac spine, posteriorsuperior iliac spine, medial and lateral femoral condyles, apex of the patella, tibial tuberosity, dorsum, medial and lateral malleoli, fifth metatarsal head, calcanei, and sacrum. Rigid clusters of four reflective markers each were placed on the subject's lateral side of the left and right thigh and shank. Figure 2-1 shows the musculoskeletal model and relative positions of the retroreflective markers.

The staircase (AMTI, Inc., Watertown, MA, USA) used in this study consisted of three steps of $28 \mathrm{~cm}$ depth, $17.8 \mathrm{~cm}$ height, and $61.0 \mathrm{~cm}$ width. It was bolted on two 3D force platforms (AMTI, Inc., Watertown, MA, USA) connected to a USB analog acquisition interface and computer running Qualysis Track Manager software (Qualisys $\mathrm{AB}$, Gothenburg, Sweden), which allowed for the collection of synchronized ground reaction force $(\mathrm{GRF})$ data during the trials.

Subjects completed a quiet standing trial prior to the testing to establish an anatomical model. Subjects were asked to start walking from two to three steps away from the staircase after receiving a signal to begin the trial. They were instructed to ascend the steps in a step-over-step manner at a self-selected pace, turn around on the elevated platform, then descend from the platform down the steps and return to the starting location. The starting leg and stride length were not specified, and subjects were allowed practice trials and rest as necessary. The test was repeated until six successful trials were acquired and three of the six were used in this study's comparison. The three 
Table 2-1. Subject metrics: mean (SD)

\begin{tabular}{lccc}
\hline \multicolumn{1}{c}{ Metric } & ACLR & Normal & p-Value \\
\hline Age (years) & $28.0(6.0)$ & $27.2(8.1)$ & 0.67 \\
Height $(\mathrm{m})$ & $1.76(0.10)$ & $1.76(0.12)$ & 0.98 \\
Body Mass (kg) & $77.5(16.7)$ & $78.3(19.6)$ & 0.90 \\
Body Mass Index $\left(\mathrm{kg} / \mathrm{m}^{2}\right)$ & $24.8(3.6)$ & $24.8(4.2)$ & 0.97 \\
Gender & $10 \mathrm{R} 5 \mathrm{~L}$ & $10 \mathrm{R} 5 \mathrm{~L}$ & $\mathrm{n} / \mathrm{a}$ \\
Reconstructed/Matched Limb & $9 \mathrm{M} \mathrm{6F}$ & $9 \mathrm{M} 6 \mathrm{~F}$ & n/a \\
\hline
\end{tabular}

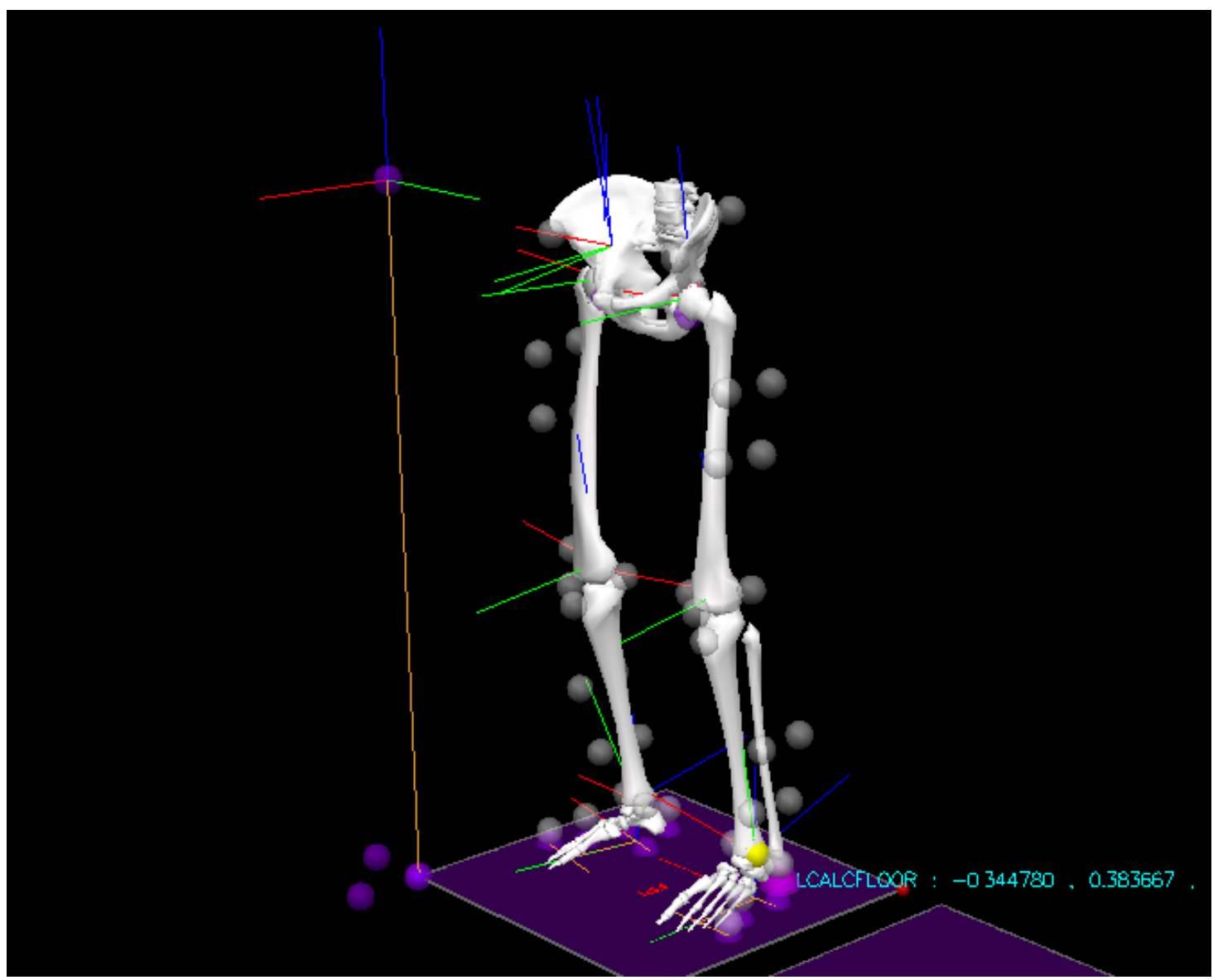

Figure 2-1. Locations of the retroreflective markers relative to the bony anatomy of the lower body 
trials with the smallest gaps in marker and ground reaction force data and no data gaps of more than ten continuous frames were selected for use in the study.

Data were analyzed by exporting the retroreflective marker trajectories and GRF data to Visual3D (C-Motion, Germantown, MD, USA). A three-dimensional musculoskeletal model was constructed using the quiet standing trial. The GRF data were used to determine the beginning and end of stance. Initial contact during the first foot strike of the analyzed limb while in stair gait is considered the beginning of stance phase and it ends when the forefoot from the same extremity leaves the step. A foot strike event was identified when a step's force plate reading surpassed a threshold based on the subject's total body mass and was then associated with the limb that had foot markers near the top surface of the corresponding step in 3D space. Foot strikes and toe offs with incomplete force plate data were identified using a threshold for the velocity of the fifth metatarsal marker based on data gathered from correctly GRF-identified foot strikes. Stance was then broken down into two sub-phases for analysis, which is intended to increase the sensitivity of the measurement as suggested by Heiderscheit et al [50]. The transition into the second phase was characterized by a change in the velocity of the center of mass. These sub-phases and the determination of the transition point were selected based on the characteristics and definitions of the phases of stair ambulation as described by Zachazewski et al [23]. Figure 1-2 shows an illustration of the two subphases used in this study. In the first segment of the descent stride, the subject moves forward toward the next step. It contains the weight acceptance and forward continuance (FC) portions of stair descent and is henceforth referred to as the FC sub-phase. This subphase concludes when the subject's center of mass velocity (VCOM) drops below zero, which signifies the transition into the controlled lowering (CL) sub-phase. Once the subject has lowered their weight onto the next step and the ipsilateral foot leaves the step, the second sub-phase ends. The amount of time spent in each sub-phase is dependent on the subject's self-selected gait speed and gait patterns, thus it was different for each subject.

A custom Matlab (MathWorks, Massachusetts, USA) script was used to calculate the sub-phase transitions and coupling angles based on the VCOM and angle data measured in Visual3D respectively. The script can be viewed in Appendix A. The Circular Statistics Toolbox from Mathworks was used to find the coupling angle variability for each subject for various combinations of joints and degrees of freedom. Mean coupling angles for the ACLR and control groups were calculated as well. Coupling angle was calculated using the following formula based on the vector coding technique described by Sparrow et al [35] and Heiderscheit et al [50]:

$$
C A=\tan ^{-1}\left(\frac{\Theta_{1, t+1}-\Theta_{1, t}}{\Theta_{2, t+1}-\Theta_{2, t}}\right)
$$

Where CA is the coupling angle, the first subscript indicates one of the two joint and plane combinations, and $t$ is the normalized time point, which is essentially representative of the percentage of stride. For all coupling abbreviations including intrajoint, the first joint and degree of freedom combination indicates $\Theta_{2}$ and the second 
indicates $\Theta_{1}$. Five couplings were selected to compare between the two groups over each sub-phase: two intra-joint knee couplings and three hip-knee couplings. The two intrajoint couplings compared were knee flexion/extension-knee abduction/adduction (KF$\mathrm{KA}$ ) and knee IE rotation/knee abduction-adduction (KR-KA). Inter-joint couplings focused on a single anatomical plane. Knee-hip sagittal (KF-HF), transverse (KR-HR), and coronal (KA-HA) plane couplings were compared to analyze the flexion/extension, rotational, and abduction/adduction joint coordination respectively. The standard deviation of the coupling angles for each of these is presented as well as the average coupling angle. The magnitude of the coupling angle has been used to identify the behavior of the coupling as defined by Chang et al [51]. These researchers used this classification to describe whether the couples are moving in opposing directions (antiphase), together (in-phase), or whether one particular joint and degree of freedom is responsible for a majority of the angular displacement.

Statistical analysis of the variability data was performed using a Wilcoxon signedrank test to compare the group mean variability of the ACLR subjects and control group for each of the selected couplings and sub-phases for a total of ten comparisons. Cohen's $d$ is used to measure the effect size of the measured differences and the $95 \%$ confidence interval is included.

\section{Results}

Data from the reconstructed subjects were compared with the control group and separated by sub-phase. Knee flexion/extension-hip flexion/extension (KF-HF) had the largest effect size of the comparisons made in the FC sub-phase with an average group variability of $5.7^{\circ}$ for the ACL group versus $8.9^{\circ}$ for the control group $(d=0.85, p=$ $0.064)$. Other knee-hip coupling variabilities in the transverse and coronal planes were not found to be different from the normal group $(d=0.23, p=0.359$ and $d=0.06, p=$ 0.890). Intra-joint coupling variabilities were also not significantly different for the reconstructed group $(d=0.36, p=0.169$ and $d=0.13, p=0.890)$. Results for these comparisons can be found in Table 2-2.

Effect sizes were larger during the CL sub-phase for four out of the five couplings and are shown in Table 2-3. In this portion of the stride, knee-hip IE rotation and abduction/adduction variabilities were smaller for the ACL group when compared with the control group $(d=1.00, p=0.0067$ and $d=0.63, p=0.083$ respectively). Average variability in the flexion/extension coupling was relatively small for both groups, though the ACLR group's was about 53\% greater. Knee IE rotation-knee abduction/adduction was lower for ACLR subjects $(\mathrm{d}=0.82)$ while the knee flexion/extension-knee abduction/adduction coupling was larger than that of the control group with a slightly lower effect size $(\mathrm{d}=0.72)$.

The group ensemble average coupling angles and their classifications are displayed in Table 2-4 and Table 2-5. As previously mentioned, the classification listed 
Table 2-2. Joint coordination variability during FC: mean (SD)

\begin{tabular}{|c|c|c|c|c|c|c|c|}
\hline \multirow[t]{2}{*}{ Coupling } & \multirow[t]{2}{*}{$\operatorname{ACLR}\left(^{\circ}\right)$} & \multirow[t]{2}{*}{ Normal $\left({ }^{\circ}\right)$} & \multirow[t]{2}{*}{ p-Value } & \multirow[t]{2}{*}{ Cohen's d } & \multicolumn{2}{|c|}{$\begin{array}{c}\text { Cohen's d } \\
\text { Confidence Interval } \\
(95 \%) \\
\end{array}$} & \multirow[t]{2}{*}{ Power } \\
\hline & & & & & Lower & Upper & \\
\hline KF-KA & $13.3(10.9)$ & $10.1(5.8)$ & 0.1688 & 0.36 & -0.37 & 1.07 & 0.16 \\
\hline KR-KA & $20.0(13.7)$ & $17.8(18.5)$ & 0.8904 & 0.13 & -0.59 & 0.85 & 0.06 \\
\hline KF-HF & $5.7(3.0)$ & $8.9(4.4)$ & 0.0637 & -0.85 & -1.58 & -0.09 & 0.61 \\
\hline KR-HR & $20.2(10.7)$ & $17.2(15.1)$ & 0.3591 & 0.23 & -0.49 & 0.94 & 0.09 \\
\hline KA-HA & $15.7(13.9)$ & $16.6(14.2)$ & 0.8904 & -0.06 & -0.77 & 0.66 & 0.05 \\
\hline
\end{tabular}

Coupling names are presented as an amalgamation of two joint and motion combinations (i.e. $\mathrm{KF}-\mathrm{KA}=\mathrm{knee}$ flexion/extension-knee abduction/adduction). $\mathrm{K}=\mathrm{knee}, \mathrm{H}=\mathrm{hip}$, $\mathrm{F}=$ flexion/extension, $\mathrm{R}=$ rotation, $\mathrm{A}=$ abduction/adduction, $\mathrm{FC}=$ forward continuance.

Table 2-3. Joint coordination variability during CL: mean (SD)

\begin{tabular}{|c|c|c|c|c|c|c|c|}
\hline \multirow[t]{2}{*}{ Coupling } & \multirow[t]{2}{*}{$\operatorname{ACLR}\left(^{\circ}\right)$} & \multirow[t]{2}{*}{ Normal $\left(^{\circ}\right)$} & \multirow[t]{2}{*}{ p-Value } & \multirow[t]{2}{*}{ Cohen's d } & \multicolumn{2}{|c|}{$\begin{array}{c}\text { Cohen's d } \\
\text { Confidence Interval } \\
(95 \%) \\
\end{array}$} & \multirow[t]{2}{*}{ Power } \\
\hline & & & & & Lower & Upper & \\
\hline KF-KA & $3.6(1.8)$ & $2.5(1.3)$ & 0.0730 & 0.72 & -0.03 & 1.44 & 0.48 \\
\hline KR-KA & $14.5(8.3)$ & $24.6(15.3)$ & 0.0554 & -0.82 & -1.54 & -0.06 & 0.58 \\
\hline KF-HF & $3.8(2.5)$ & $2.5(1.5)$ & 0.1070 & 0.64 & -0.11 & 1.35 & 0.39 \\
\hline KR-HR & $17.5(14.4)$ & $34.9(20.0)$ & 0.0067 & -1.00 & -1.73 & -0.21 & 0.75 \\
\hline KA-HA & $14.7(14.7)$ & $23.2(12.4)$ & 0.0833 & -0.63 & -1.34 & 0.12 & 0.38 \\
\hline
\end{tabular}

Coupling names are presented as an amalgamation of two joint and motion combinations (i.e. KF-KA=knee flexion/extension-knee abduction/adduction). $\mathrm{K}=\mathrm{knee}, \mathrm{H}=\mathrm{hip}$, $\mathrm{F}=$ flexion/extension, $\mathrm{R}=$ rotation, $\mathrm{A}=$ abduction/adduction, $\mathrm{CL}=$ controlled lowering. 
Table 2-4. Coordination phase magnitude and classification during FC: mean (classification)

\begin{tabular}{lcc}
\hline \multicolumn{1}{c}{ Coupling } & ACLR $\left({ }^{\circ}\right)$ & Normal $\left({ }^{\circ}\right)$ \\
\hline KF-KA & $96(\mathrm{KF})$ & $88(\mathrm{KF})$ \\
KR-KA & $91(\mathrm{KR})$ & $87(\mathrm{KR})$ \\
KF-HF & 135 (Anti-phase) & 124 (Anti-phase) \\
KR-HR & $81(\mathrm{KR})$ & $76(\mathrm{KR})$ \\
KA-HA & $3(\mathrm{HA})$ & $20(\mathrm{HA})$ \\
\hline
\end{tabular}

Coupling names are presented as an amalgamation of two joint and motion combinations (i.e. KF-KA=knee flexion/extension-knee abduction/adduction). $\mathrm{K}=\mathrm{knee}, \mathrm{H}=\mathrm{hip}$, $\mathrm{F}=$ flexion/extension, $\mathrm{R}=$ rotation, $\mathrm{A}=$ abduction/adduction, $\mathrm{FC}=$ forward continuance.

Table 2-5. Coordination phase magnitude and classification during CL: mean (classification)

\begin{tabular}{lcc}
\hline \multicolumn{1}{c}{ Coupling } & ACLR $\left(^{\circ}\right)$ & Normal $\left(^{\circ}\right)$ \\
\hline KF-KA & $82(\mathrm{KF})$ & $86(\mathrm{KF})$ \\
KR-KA & $96(\mathrm{KR})$ & $130($ Anti-phase) \\
KF-HF & $87(\mathrm{KF})$ & $86(\mathrm{KF})$ \\
KR-HR & 48 (In-phase) & $112(\mathrm{KR})$ \\
KA-HA & $81(\mathrm{KA})$ & $91(\mathrm{KA})$ \\
\hline
\end{tabular}

Coupling names are presented as an amalgamation of two joint and motion combinations (i.e. KF-KA=knee flexion/extension-knee abduction/adduction). $\mathrm{K}=\mathrm{knee}, \mathrm{H}=\mathrm{hip}$, $\mathrm{F}=$ flexion/extension, $\mathrm{R}=$ rotation, $\mathrm{A}=$ abduction/adduction, $\mathrm{CL}=$ controlled lowering. 
describes whether the coupling was primarily in-phase, anti-phase, or a particular joint and movement combination was dominant during the sub-phase. Our results showed no difference in coordination classification for the forward continuance sub-phase. Though variability was not necessarily the same, phase magnitude was similar for all measured couplings. The CL sub-phase revealed two differences. The knee rotation-knee abduction/adduction coupling was found to be mostly dominated by the changes in rotation angle for the ACL group whereas the control group coupling angle indicated anti-phase coordination. Knee rotation-hip rotation was also different in that rotation was primarily seen in the knee for the healthy group while the ACLR subjects' hip and knee rotated in phase. Phase magnitude calculations and classifications for the FC sub-phase are displayed in Table 2-4, and results for the CL sub-phase are shown in Table 2-5.

\section{Discussion}

The purpose of this study was to identify differences during stair descent in the coupling angle variability of subjects who had undergone unilateral anterior cruciate ligament reconstruction surgery as compared with subjects with healthy lower extremities. Our hypothesis was that subjects' movement patterns would be altered to adapt to the graft to complete the stairs task and that these adaptations would be reflected in the variability measurement as compared with the control group. This hypothesis was not supported by the data after Bonferroni correction for the ten selected coupling comparisons. The study design was exploratory in nature and had insufficient power (ranging from 0.05 to 0.75 ) to permit definitive conclusions to be drawn. However, several of the data comparisons yielded a large effect size and could be significant with a more focused or less conservative approach. In particular, the knee rotation-hip rotation coupling displayed a much lower ensemble-average variability for the affected group $(\mathrm{p}=$ 0.0067) during the controlled lowering sub-phase of stance.

If there are, in fact, changes to the ability of the knee to adaptively rotate they may be related to aspects of the reconstructed ACL graft such as graft type and/or placement. The native ACL consists of two bundles - the anteromedial (AM) and posterolateral (PL) bundles. While the two bundles of the ACL work together to limit anterior tibial translation and add stability to rotation of the knee, each band is under a different amount of tension depending on the flexion angle of the knee. During the forward continuance portion of stance, the knee is extended, while during the controlled lowering sub-phase the knee is flexed to a maximum of about 77 degrees to lower the body on to the next step and transition to swing phase [17]. At this degree of flexion a healthy limb would be more reliant upon the AM bundle for stability while the PL bundle would be more lax. The majority of results with large effect sizes in this study were found during the second portion of stance, indicating more overall altered variability when the leg is more flexed. Researchers have previously theorized that, because an ACL autograft is typically harvested from the more uniform patellar tendon, it's functionality would logically be more representative of single bundle anatomy [29]. This line of reasoning can be extended to those subjects having received a hamstring autograft as 
well, implying that these graft types might not be capable of providing the dynamic stability of the dual-banded ACL in all levels of knee flexion.

Previous studies on walking, jogging, and cutting have indicated that ACLR subjects show increased joint coordination variability and that this could be an indicator of reduced stability in the knee $[46,47,48]$. In this study, differences between variabilities during the FC part of stance phase were relatively small with the exception of the KF-HF coupling, which had a medium effect size. Most of the forward continuance portion of stair descent is similar to walking since the subject has not yet begun to lower their bodyweight to the next step. Therefore, it is not surprising that our findings agree with those of Gribbin et al who found very few differences in early stance of walking gait. Contrary to findings during walking and jogging activities by researchers Davis et al [46] and Gribbin et al [47], the variabilities for our affected subjects' KR-HR and KA-HA couplings were lower than those of the controls $(0.05<p<0.10)$. These previous researchers found significantly greater variability in both couplings. Pollard et al [48] were the only research group to investigate intra-joint coordination prior to this study. We found differences with a medium effect size in both of the measured intra-joint coupling variabilities during the CL sub-phase. KF-KA was greater for the ACL group $(d=0.72, p$ $=0.073)$ and KR-KA was lower $(d=0.82, p=0.055)$, whereas Pollard's observations were that both variabilities were increased for reconstructed subjects during a cutting maneuver.

The reasons for the discrepancy in the direction of the difference for this particular task are unknown, but Pollard et al [52] have presented data that correlate reduced variability in ACLR subjects with increased injury risk. Noting that females are several times more likely to sustain a knee injury compared with males in the same sport, they compared male and female reconstruction patients and found that four of the observed couplings showed lower variability for the female group. Reduced variability in this case could represent a decreased ability of the subject to adapt to a dynamic locomotory task. Lower variability has also been connected with knee pain, and it is suggested that this may be a sign of the subject protecting the affected joint $[8,44]$.

It is important to note the limitations in our study. Of our fifteen subjects, eleven had received a bone-patellar-tendon-bone autograft, one had a hamstring graft, two received cadaveric allografts, and one was unknown. There are likely functional differences among these graft types. Also, the retroreflective marker tracking method has the disadvantage of increased soft tissue artifact for subjects with a high body mass index, which may have contributed error to these data, particularly for the hip. Despite our exclusion criterion of an upper limit for BMI, some of our subjects were classified as overweight or obese. Lastly, only the proximal couplings were considered, and we determined the ankle to be outside the scope of this study. Future work may consider analyzing knee-ankle and hip-ankle coupling angles, especially since the latter has been found to be important in a previous work that studied the variability of joint coordination during a balancing task [2]. Other, mixed planar couplings related to the types of motion associated with ACL injury could also be investigated. 
In conclusion, our study did not find strictly significant differences in the variability of joint coordination between the observed groups, but many of the measured differences exhibited a large calculated effect size. We believe that a more focused approach with these comparisons may lead to discoveries of aberrant coordination in ACL subjects and that these differences should be explored as possible indicators of reinjury risk. 


\section{CHAPTER 3. ADDITIONAL RESULTS FOR OMITTED VARIABLES}

\section{Chapter Overview}

The previous chapter's focus was narrowed to only make the comparisons that we hypothesized would be different based on previous studies' results. The stair ascent task, contralateral limbs, and ankle joint kinematics were tracked during the original collection of the data. In this chapter, notable differences in these categories are reported. The results are briefly discussed in the context of what variables may be of interest for future research with the acknowledgement that a complete statistical analysis is necessary to draw conclusions. The results shown in this chapter may be useful to other researchers who are interested in investigating these other variables and suggest what joint couplings to focus on.

\section{Results}

\section{ACLR versus Healthy Control}

The results provided in Table 3-1 show comparisons between reconstructed limbs and normal matched limbs that had at least a moderate effect size during the ascent task. Interestingly, all of these results show decreased variability and occur during the pull-up (PU) sub-phase of the task. There were no notable differences during the FC sub-phase of ascent, and a complete table of calculated joint coordination variability for all measured couplings can be seen in Appendix B (Table B-1). The FC sub-phase of descent also saw fewer notable effect sizes. Selected coupling variabilities observed during descent are shown in Table 3-2. Most of these results are previously discussed in Chapter 2 and the complete set of average joint coordination variabilities for the descent task are shown in (Table B-2). Several couplings involving ankle dorsi/plantarflexion may be different as compared with the control group. The ankle was omitted from the statistical analysis because there was little precedent found in the review of literature and previously mentioned work by Davis et al [46] found very small differences during walking and to limit the number of comparisons in the interest of mitigating the possibility of falsely rejecting the null hypothesis that there was no difference. We also did not have confidence that the foot and ankle model used in the musculoskeletal model was fully representative of the segment. The range of ankle dorsi/plantarflexion angles on stairs is considerably larger than it is during walking, with the ascent portion of the activity displaying the larger range. One article by Paterno et al [2] linked reduced sagittal plane hip-ankle coupling variability during a balancing task with re-injury risk for ACL subjects. Their reason for evaluation of this specific coupling was based on their review of proprioception studies from which they concluded that the knee's loss of proprioception due to the loss of the native ACL would result in compensation by the proximal and distal joints. A study with a scope that includes balance and proprioception might be able to make use of these data. 
Table 3-1. Reconstructed limb versus normal matched limb - joint coordination variability during stair ascent: mean (SD)

\begin{tabular}{|c|c|c|c|c|c|c|}
\hline \multirow{2}{*}{$\begin{array}{c}\text { Stance } \\
\text { Sub- } \\
\text { Phase }\end{array}$} & \multirow[t]{2}{*}{ Coupling } & \multirow{2}{*}{$\begin{array}{c}\text { ACLR } \\
\left({ }^{\circ}\right)\end{array}$} & \multirow{2}{*}{$\begin{array}{l}\text { Normal } \\
\left({ }^{\circ}\right)\end{array}$} & \multirow[t]{2}{*}{ Cohen's d } & \multicolumn{2}{|c|}{$\begin{array}{c}\text { Confidence Interval } \\
(\mathbf{9 5 \%})\end{array}$} \\
\hline & & & & & Lower & Upper \\
\hline PU & KF-HF & $3.3(2.9)$ & $5.7(3.3)$ & -0.80 & -1.54 & -0.06 \\
\hline PU & KR-AF & $\begin{array}{c}21.4 \\
(16.7)\end{array}$ & $\begin{array}{c}32.1 \\
(14.4)\end{array}$ & -0.68 & -1.42 & 0.05 \\
\hline PU & KR-HR & $\begin{array}{c}18.4 \\
(15.1)\end{array}$ & $\begin{array}{c}31.9 \\
(21.7)\end{array}$ & -0.72 & -1.46 & 0.02 \\
\hline PU & KR-HA & $11.7(9.3)$ & $17.5(9.4)$ & -0.62 & -1.35 & 0.12 \\
\hline PU & AF-HF & $4.5(3.1)$ & $6.7(4.1)$ & -0.60 & -1.33 & 0.14 \\
\hline PU & AF-HA & $\begin{array}{c}16.7 \\
(12.5)\end{array}$ & $\begin{array}{c}25.9 \\
(17.8) \\
\end{array}$ & -0.55 & -1.28 & 0.18 \\
\hline
\end{tabular}

Coupling names are presented as an amalgamation of two joint and motion combinations (i.e. $\mathrm{KF}-\mathrm{KA}=\mathrm{knee}$ flexion/extension-knee abduction/adduction). $\mathrm{K}=\mathrm{knee}, \mathrm{H}=\mathrm{hip}$, $\mathrm{F}=$ flexion/extension, $\mathrm{R}=$ rotation, $\mathrm{A}=$ abduction/adduction, $\mathrm{PU}=$ pull up. 
Table 3-2. Reconstructed limb versus normal matched limb - joint coordination variability during stair descent: mean (SD)

\begin{tabular}{|c|c|c|c|c|c|c|}
\hline \multirow{2}{*}{$\begin{array}{c}\text { Stance } \\
\text { Sub- } \\
\text { Phase }\end{array}$} & \multirow[t]{2}{*}{ Coupling } & \multirow{2}{*}{$\begin{array}{c}\text { ACLR } \\
\left({ }^{\circ}\right)\end{array}$} & \multirow{2}{*}{$\begin{array}{l}\text { Normal } \\
\left({ }^{\circ}\right)\end{array}$} & \multirow[t]{2}{*}{ Cohen's d } & \multicolumn{2}{|c|}{$\begin{array}{c}\text { Confidence Interval } \\
(\mathbf{9 5 \%})\end{array}$} \\
\hline & & & & & Lower & Upper \\
\hline FC & KF-HF & $5.7(3.0)$ & $8.9(4.4)$ & -0.85 & -1.60 & -0.11 \\
\hline $\mathrm{FC}$ & KF-HR & $16.1(9.4)$ & $11.4(6.0)$ & 0.59 & -0.14 & 1.32 \\
\hline $\mathrm{FC}$ & KR-AF & $8.9(8.4)$ & $5.4(3.0)$ & 0.57 & -0.16 & 1.29 \\
\hline $\mathrm{FC}$ & AF-HR & $8.5(4.0)$ & $5.9(3.6)$ & 0.67 & -0.06 & 1.41 \\
\hline CL & KR-HR & $\begin{array}{c}17.5 \\
(14.4)\end{array}$ & $\begin{array}{c}34.9 \\
(20.0)\end{array}$ & 1.00 & -1.75 & -0.24 \\
\hline CL & KR-KA & $14.5(8.3)$ & $\begin{array}{c}24.6 \\
(15.3)\end{array}$ & -0.82 & -1.57 & -0.08 \\
\hline CL & HR-HA & $\begin{array}{c}19.5 \\
(17.0)\end{array}$ & $\begin{array}{c}32.8 \\
(14.5)\end{array}$ & -0.84 & -1.59 & -0.09 \\
\hline CL & KF-HF & $3.8(2.5)$ & $2.5(1.5)$ & 0.64 & -0.10 & 1.37 \\
\hline $\mathrm{CL}$ & KF-KA & $3.6(1.8)$ & $2.5(1.3)$ & 0.72 & -0.02 & 1.46 \\
\hline $\mathrm{CL}$ & KA-HR & $\begin{array}{c}15.7 \\
(13.6)\end{array}$ & $\begin{array}{l}29.1 \\
(20.5)\end{array}$ & -0.77 & -1.51 & -0.03 \\
\hline CL & KA-HA & $\begin{array}{c}14.7 \\
(14.7)\end{array}$ & $\begin{array}{l}23.2 \\
(12.4)\end{array}$ & -0.63 & -1.36 & 0.11 \\
\hline CL & AF-HR & $\begin{array}{c}12.9 \\
(16.1)\end{array}$ & $\begin{array}{l}24.9 \\
(20.1)\end{array}$ & -0.66 & -1.39 & 0.08 \\
\hline CL & AF-HA & $\begin{array}{c}11.4 \\
(10.3)\end{array}$ & $\begin{array}{c}20.8 \\
(15.3)\end{array}$ & -0.73 & -1.47 & 0.01 \\
\hline $\mathrm{CL}$ & HF-HR & $11.2(5.2)$ & $14.6(5.6)$ & -0.64 & -1.37 & 0.09 \\
\hline
\end{tabular}

Coupling names are presented as an amalgamation of two joint and motion combinations (i.e. $\mathrm{KF}-\mathrm{KA}=\mathrm{knee}$ flexion/extension-knee abduction/adduction). $\mathrm{K}=\mathrm{knee}, \mathrm{H}=\mathrm{hip}$, $\mathrm{F}=$ flexion/extension, $\mathrm{R}=$ rotation, $\mathrm{A}=$ abduction/adduction, $\mathrm{FC}=$ forward continuance, $\mathrm{CL}=$ controlled lowering. 


\section{Contralateral Limb}

Some studies have investigated the contralateral limbs of ACLR subjects using similar methods or traditional kinematics measurements. Of the studies reviewed for this work, only Ristanis et al [32] made comparisons between the contralateral limbs of ACLR subjects and those of healthy control subjects. They did not find differences between the groups when comparing kinematics. There were several within subject studies that compared the reconstructed leg to the contralateral. The results for these studies were generally very similar to those previously discussed comparing reconstructed limbs with the limbs of control subjects. Two studies using dynamical systems methods found greater variability in the sagittal plane $[53,54]$ and two articles on kinematics reported differences in maximum knee extension, internal and external tibial rotation, and tibial adduction $[55,56]$. The discussions presented by these studies assumed that the uninjured leg of the ACLR subjects was not impacted by changes in the limb with the reconstructed ACL. Nevertheless, it may still be possible that a compromised ACL in one limb affects the opposite extremity as a compensatory mechanism for the ipsilateral's altered joint kinematics or joint coordination variability. Notable ascent task results for comparisons between the reconstructed and contralateral limbs from our data are shown in Table 3-3, and comparisons between the contralateral limb and matched normal contralateral limb are displayed in Table 3-4. Most of the moderate and large differences were in the FC sub-phase of Ascent. Also, the reconstructed limb generally had more variability. Descent results comparing the reconstructed with the contralateral are in Table 3-5. The contralateral limb variability as compared with the normal matched contralateral did not produce any differences classified as moderate or large during the stair descent activity.

\section{Swing Phase}

Studies that performed kinematics or joint coordination analyses detected very few differences during swing phase $[47,55]$. The vast majority of reviewed studies omitted the swing phase entirely. It is possible that differences in the reconstructed subjects are more apparent when the leg is bearing a load. Nevertheless, stair swing phase is different from other activities such as walking and jogging because it is composed of the foot placement and foot clearance activities during both ascending and descending. Swing phase was ultimately deemed outside of the scope of work of this research though it may be of interest to others, particularly those interested in the foot positioning aspects which might be affected by altered proprioception. 
Table 3-3. Reconstructed limb versus contralateral limb - joint coordination variability during stair ascent: mean (SD)

\begin{tabular}{llccccc}
\hline $\begin{array}{c}\text { Stance } \\
\text { Sub- } \\
\text { Phase }\end{array}$ & Coupling & ACLR $\left(^{\circ}\right)$ & ACLC ( $\left(^{\circ}\right)$ & Cohen's d & \multicolumn{2}{c}{$\begin{array}{c}\text { Confidence Interval } \\
\text { (95\%) }\end{array}$} \\
\cline { 6 - 7 } PU & KF-AF & $5.1(3.3)$ & $3.3(3.1)$ & 0.57 & -0.16 & Upper \\
PU & AF-HA & 16.7 & $10.9(6.4)$ & 0.59 & -0.14 & 1.30 \\
& & $(12.5)$ & & & & \\
FC & KF-HF & 17.3 & $10.5(4.7)$ & 0.68 & -0.05 & 1.42 \\
& & $(13.3)$ & & & -0.22 & 1.23 \\
FC & KF-HR & $(25.4)$ & $(17.4)$ & & & \\
& & 25.3 & 16.9 & 0.60 & -0.13 & 1.33 \\
FC & KF-HA & $(16.0)$ & $(11.7)$ & & & \\
& & 39.1 & 26.0 & 0.65 & -0.08 & 1.39 \\
FC & KF-KA & $(22.4)$ & $(17.2)$ & & & \\
& & 17.7 & $8.9(5.9)$ & 0.67 & -0.07 & 1.40 \\
FC & HF-HR & $(17.5)$ & & & & \\
\hline
\end{tabular}

Coupling names are presented as an amalgamation of two joint and motion combinations (i.e. $\mathrm{KF}-\mathrm{KA}=$ knee flexion/extension-knee abduction/adduction). $\mathrm{K}=\mathrm{knee}, \mathrm{H}=$ hip, $\mathrm{F}=$ flexion/extension, $\mathrm{R}=$ rotation, $\mathrm{A}=$ abduction/adduction, $\mathrm{PU}=$ pull up, $\mathrm{FC}=$ forward continuance. 
Table 3-4. Contralateral limb versus normal matched contralateral limb - joint coordination variability during stair ascent: mean (SD)

\begin{tabular}{llccccc}
\hline $\begin{array}{c}\text { Stance } \\
\begin{array}{c}\text { Sub- } \\
\text { Phase }\end{array}\end{array}$ & Coupling & $\begin{array}{c}\text { ACLC } \\
\left({ }^{\circ}\right)\end{array}$ & $\begin{array}{c}\text { NormalC } \\
\left({ }^{\circ}\right)\end{array}$ & Cohen's d & \multicolumn{2}{c}{$\begin{array}{c}\text { Confidence Interval } \\
(\mathbf{9 5 \% )})\end{array}$} \\
\hline PU & AF-HF & $3.3(2.8)$ & $4.8(1.7)$ & -0.65 & -1.39 & 0.08 \\
FC & KA-AF & 18.4 & $8.7(5.2)$ & 0.86 & 0.11 & 1.60 \\
& KF-HF & $10.5(4.7)$ & $17.2(15.9)$ & -0.58 & -1.31 & 0.15 \\
FC & KR-AF & 20.1 & $12.2(8.1)$ & 0.51 & -0.21 & 1.24 \\
FC & & $(20.4)$ & & & \\
& AF-HR & $(18.7$ & $8.8(7.7)$ & 0.72 & -0.02 & 1.46 \\
FC & & $(18.0)$ & & & \\
& HF-HR & $8.9(5.9)$ & $20.0(19.1)$ & -0.78 & -1.52 & -0.04 \\
FC & HF-HA & $6.5(3.8)$ & $9.2(5.6)$ & -0.56 & -1.29 & 0.17 \\
FC & & & & & & \\
\hline
\end{tabular}

Coupling names are presented as an amalgamation of two joint and motion combinations (i.e. $\mathrm{KF}-\mathrm{KA}=\mathrm{knee}$ flexion/extension-knee abduction/adduction). $\mathrm{K}=\mathrm{knee}, \mathrm{H}=\mathrm{hip}$, $\mathrm{F}=$ flexion/extension, $\mathrm{R}=$ rotation, $\mathrm{A}=$ abduction/adduction, $\mathrm{PU}=$ pull up, $\mathrm{FC}=$ forward continuance. 
Table 3-5. Reconstructed limb versus contralateral limb - joint coordination variability during stair descent: mean (SD)

\begin{tabular}{llccccc}
\hline $\begin{array}{c}\text { Stance } \\
\text { Sub- } \\
\text { Phase }\end{array}$ & Coupling & \multirow{2}{*}{$\begin{array}{c}\text { ACLR } \\
\left({ }^{\circ}\right)\end{array}$} & $\begin{array}{c}\text { ACLC } \\
\left({ }^{\circ}\right)\end{array}$ & Cohen's d & \multicolumn{2}{c}{$\begin{array}{c}\text { Confidence Interval } \\
\mathbf{( 9 5 \% )}\end{array}$} \\
\cline { 6 - 7 } & & & & & Lower & Upper \\
FC & KF-HF & $5.7(3.0)$ & $7.8(4.2)$ & -0.57 & -1.30 & 0.16 \\
FC & AF-HR & $8.5(4.0)$ & $5.6(3.8)$ & 0.75 & 0.01 & 1.49 \\
FC & AF-HA & $7.3(7.0)$ & $4.2(3.3)$ & 0.58 & -0.15 & 1.31 \\
CL & KF-HR & $4.7(2.5)$ & $3.5(1.8)$ & 0.55 & -0.18 & 1.27 \\
CL & KF-KR & $4.8(2.9)$ & $3.2(1.7)$ & 0.66 & -0.08 & 1.39 \\
& KR-HA & 21.6 & 31.1 & -0.59 & -1.32 & 0.14 \\
CL & & $(12.9)$ & $(18.8)$ & & & \\
& KR-KA & $14.5(8.3)$ & 24.6 & -0.67 & -1.40 & 0.07 \\
\hline
\end{tabular}

Coupling names are presented as an amalgamation of two joint and motion combinations (i.e. $\mathrm{KF}-\mathrm{KA}=\mathrm{knee}$ flexion/extension-knee abduction/adduction). $\mathrm{K}=\mathrm{knee}, \mathrm{H}=\mathrm{hip}$, $\mathrm{F}=$ flexion/extension, $\mathrm{R}=$ rotation, $\mathrm{A}=$ abduction/adduction, $\mathrm{FC}=$ forward continuance, $\mathrm{CL}=$ controlled lowering. 


\section{CHAPTER 4. GENERAL DISCUSSION}

\section{Introduction}

The goal of this research was to determine differences between ACLR subjects and a control group during stair ambulation. We performed the test using motion capture and ground reaction force measuring equipment while the subjects climbed a fixed staircase. Kinematics data were analyzed using the Visual3D program (C-Motion, Germantown, MD, USA) and a custom Matlab (MathWorks, Massachusetts, USA) script was employed to perform coupling angle calculations. The dependent variables in our experiment were the sub-phase of gait, the coupling angle variability of ten joint couplings, and the presence of a unilateral ACL graft.

Our hypothesis that there would be altered joint coordination variability during the descent portion of stair traversal was not supported when a correction for multiple comparisons was applied. Furthermore, the statistical power of these comparisons was below the commonly suggested $80 \%$, thus there is an unacceptably high risk of failing to reject the null hypothesis with this number of subjects. Several couplings displayed differences that could be more notable in a study with fewer variables or a task that is more difficult to complete. The results of this study do not conclusively say that there are significant differences in the dependent variables compared but they indicate that further investigations with modifications to the study design may be warranted.

\section{Limitations and Assumptions}

\section{Limitations}

- We elected not to analyze internal/external rotation and abduction/adduction of the ankle because we used a single segment model for the foot and did not deem it to be accurately representative of the subjects' anatomy.

- The study was limited to subjects with a BMI below 35 but soft tissue artefacts may still exist for subjects near this cutoff, especially at the pelvis since this is a common location for fat storage.

- ACLR subjects did not all receive the same graft type and one was unknown.

- Shapiro-Wilks tests for normality did not show that the data was normally distributed.

- Comorbidities were present in several ACLR subjects such as meniscus, hamstring, and medial collateral ligament injury or tear.

- The number of subjects available for use in this study was not sufficient to achieve $80 \%$ power for any of the comparisons made. Therefore, this work has a greater possibility for false negatives than is typically desired.

- The number of comparisons made with the same data set increased the likelihood of false positives. When a Bonferroni correction was applied to the statistical 
analysis to account for the ten comparisons, there were no comparisons displaying an acceptable amount of possible Type I error when a base confidence level of $95 \%$ was used in the calculation.

\section{Addressed Limitation}

- The non-parametric Wilcoxon Signed Rank Test was used to determine the statistical significance of the data.

\section{Assumptions}

- Soft tissue artefact did not affect the kinematics measurements.

- Leg dominance was not relevant to the outcomes.

- In order for the Cohen's d method to be useful, it was assumed that the variances in the groups being compared were homogeneous.

\section{Future Work}

Future work may consider analyzing the role of proprioception in position sense as it is related to this part of the task, particularly since improper foot placement can lead to falls. This could potentially be used in combination with the swing phase data. The native ACL contains mechanoreceptors that are believed to significantly contribute to neuromuscular control during lower body movements and major adjacent joints may compensate for reduced proprioception after a reconstruction $[57,58]$.

A pivoting maneuver following a staircase or step-down activity was found to have significant results in some of the studies reviewed for this research [28,32]. Change of direction is known to be the type of motion most likely to incur an injury of the ACL $[59,60]$. Kobayashi et al previously reported that "knee-in \& toe-out" is the most common limb position during ACL injury [61]. Movements that induce knee valgus may be of interest, particularly in the knee rotation-ankle rotation coupling.

An alternate arrangement of retroreflective markers of the foot, standardized footwear that allows for better fixation, or a change in the position of the subjects relative to the cameras may be necessary to make accurate measurements in all six degrees of freedom of the current model if the ankle is to be analyzed further. The present study found that there were large gaps in the motion tracking data for some of the markers for the foot and ankle. If a study collecting data during pivoting or cutting maneuvers were to be performed, a well-defined foot and ankle model would be an important criterion for the analysis.

Further investigation into stairs may still yield interesting results. Though a comprehensive statistical analysis was not performed, the data collected in this study 
suggest fewer differences during the FC portion of gait during either climbing task as compared to the CL or PU sub-phases. A more focused approach might consider looking only at these sub-phases or separating by single and double-leg stance, rather than considering the entire stance phase. The correlation between greater activation of the quadriceps and joint coordination variability could be of interest.

\section{Conclusions}

Re-injury rate for ACLR subjects is high and calls for in-depth analysis of lower body movement to determine if aberrant movement patterns play a role. A review of the existing literature on the differences in the traditional kinematics of ACLR subjects as compared to healthy subjects showed very little consensus among researchers. Differences due to the complexity of the system may not be measurable without employing a more detailed examination method, as seen in studies using joint coordination measurements such as the vector coding method that was applied in our study. In general, most comparisons showed decreased variability, which is linked to joint position avoidance and instability. The data collected in this work also suggest that there were a greater number of notable differences during the CL sub-phase of stair gait, but these statements cannot be made with a high degree of certainty as the current statistical approach displays a possibility for falsely rejecting the null hypothesis that exceeds the standard acceptable limit. Additional studies using the vector coding method should consider tasks involving a change of direction and focus on specific sub-phases and joint couplings. 


\section{LIST OF REFERENCES}

1. Paschos, N.K. Anterior cruciate ligament reconstruction and knee osteoarthritis. World J Orthop, 2017. 8(3): p. 212-7.

2. Paterno, M.V. Incidence and predictors of second anterior cruciate ligament injury after primary reconstruction and return to sport. J Athl Train, 2015: 50(10): p. 1097-9.

3. Makhmalbaf, H., et al. Accuracy of lachman and anterior drawer tests for anterior cruciate ligament injuries. Arch Bone Jt Surg, 2013. 1(2): p. 94-7.

4. Kilinc, B.E., et al. Evaluation of the accuracy of Lachman and Anterior Drawer Tests with KT1000 in the follow-up of anterior cruciate ligament surgery. J Exerc Rehabil, 2016. 12(4): p. 363-7.

5. Wang, H., et al. Correlation among proprioception, muscle strength, and balance. J Phys Ther Sci, 2016. 28(12): p. 3468-72.

6. Relph, N., Herrington, L., Tyson, S. The effects of ACL injury on knee proprioception: a meta-analysis. Physiotherapy, 2014. 100(3): p. 187-95.

7. Ma, Y., et al. Balance ability and proprioception after single-bundle, singlebundle augmentation, and double-bundle ACL reconstruction.

ScientificWorldJournal, 2014. 342012.

8. Markatos, K., et al. The anatomy of the ACL and its importance in ACL reconstruction. Eur J Orthop Surg Traumatol, 2013. 23(7): p. 747-52.

9. Duthon, V.B., et al. Anatomy of the anterior cruciate ligament. Knee Surg Sports Traumatol Arthrosc, 2006. 14(3): p. 204-13.

10. Zantop, T., et al. The role of the anteromedial and posterolateral bundles of the anterior cruciate ligament in anterior tibial translation and internal rotation. Am J Sports Med, 2007. 35(2): p. 223-7.

11. Petersen, W., Zantop, T. Anatomy of the anterior cruciate ligament with regard to its two bundles. Clin Orthop Relat Res, 2007. 454:35-47.

12. Amis, A.A. The functions of the fibre bundles of the anterior cruciate ligament in anterior drawer, rotational laxity and the pivot shift. Knee Surg Sports Traumatol Arthrosc, 2012. 20(4): p. 613-20.

13. Otsubo, H., et al. The arrangement and the attachment areas of three ACL bundles. Knee Surg Sports Traumatol Arthrosc, 2012. 20(1): p. 127-34.

14. MacKay, J.W., Whitehead, H., Toms, A.P. Radiological evidence for the triple bundle anterior cruciate ligament. Clin Anat, 2014. 27(7): p. 1097-102.

15. Macaulay, A.A., Perfetti, D.C., Levine, W.N. Anterior Cruciate Ligament Graft Choices. Sports Health, 2012. 4(1): p. 63-8.

16. Gifstad, T., et al. Lower risk of revision with patellar tendon autografts compared with hamstring autografts: a registry study based on 45,998 primary ACL reconstructions in Scandinavia. Am J Sports Med, 2014. 42(10): p. 2319-28. 
17. Cavaignac, E., et al. Is quadriceps tendon autograft a better choice than hamstring autograft for anterior cruciate ligament reconstruction? A comparative study with a mean follow-up of 3.6 years. Am J Sports Med, 2017. 45(6): p. 132632.

18. Björnsson, H., et al. Is double-bundle anterior cruciate ligament reconstruction superior to single-bundle? A comprehensive systematic review. Knee Surg Sports Traumatol Arthrosc, 2015. 23(3): p. 696-739.

19. Li, Y.L., et al. Single-bundle or double-bundle for anterior cruciate ligament reconstruction: a meta-analysis. Knee, 2014. 21(1): p. 28-37.

20. Desai, N., et al. Anatomic single-versus double-bundle ACL reconstruction: a meta-analysis. Knee Surg Sports Traumatol Arthrosc, 2014. 22(5): p. 1009-23.

21. Lin, H-C., Lu, T-W., Hsu, H-C. Three-dimensional analysis of kinematic and kinetic coordination of the lower limb joints during stair ascent and descent. Biomed. Eng. Appl. Basis Commun, 2004. 16: p. 101-8.

22. McFadyen, B.J., Winter, D.A. An integrated biomechanical analysis of normal stair ascent and descent. J Biomech, 1988. 21(9): p. 733-44.

23. Zachazewski, J.E., Riley, P.O., Krebs, D.E. Biomechanical analysis of body mass transfer during stair ascent and descent of healthy subjects. J Rehabil Res Dev, 1993. 30(4): p. 412-22.

24. Protopapadaki, A., et al. Hip, knee, ankle kinematics and kinetics during stair ascent and descent in healthy young individuals. Clin Biomech (Bristol, Avon), 2007. 22(2): p. 203-10.

25. Lepley, A.S., et al. Longitudinal evaluation of stair walking biomechanics in patients with ACL injury. Med Sci Sports Exerc, 2016. 48(1): p. 7-15.

26. Berchuck, M., et al. Gait adaptations by patients who have a deficient anterior cruciate ligament. J Bone Joint Surg Am, 1990. 72(6): p. 871-7.

27. Gao, B., Cordova, M.L., Zheng, N.N. Three-dimensional joint kinematics of ACLdeficient and ACL-reconstructed knees during stair ascent and descent. Hum Mov Sci, 2012. 31(1): p. 222-35.

28. Claes, S., et al. Tibial rotation in single- and double-bundle ACL reconstruction: a kinematic 3-D in vivo analysis. Knee Surg Sports Traumatol Arthrosc, 2011. 19 (Suppl 1): p. S115-21.

29. Brandsson, S., et al. Kinematics and laxity of the knee joint after anterior cruciate ligament reconstruction: pre- and postoperative radiostereometric studies. Am J Sports Med, 2002. 30(3): p. 361-7.

30. Hall, M., Stevermer, C.A., Gillette, J.C. Gait analysis post anterior cruciate ligament reconstruction: knee osteoarthritis perspective. Gait Posture, 2012. 36(1): p. 56-60. 
31. Sole, G., et al. Knee kinematics during stair descent 20 years following anterior cruciate ligament rupture with and without reconstruction. Clin Biomech (Bristol, Avon), 2016. 32: p. 180-6.

32. Ristanis, S., et al. The effects of anterior cruciate ligament reconstruction on tibial rotation during pivoting after descending stairs. Knee Surg Sports Traumatol Arthrosc, 2003. 11(6): p. 360-5.

33. Spinelli, B.A., et al. Using kinematics and a dynamical systems approach to enhance understanding of clinically observed aberrant movement patterns. Man Ther, 2015. 20(1): p. 221-6.

34. Nematollahi, M., et al. Inter-segmental coordination pattern in patients with anterior cruciate ligament deficiency during a single-step descent. PLoS One, 2016. 11(2): e0149837.

35. Sparrow, W.A., et al. Using relative motion plots to measure changes in intralimb and inter-limb coordination. J Mot Behav, 1987. 19(1): p. 115-29.

36. van Emmerik R.E., van Wegen E.E. On variability and stability in human movement. J Appl Biomech, 2000. 16(4): p. 394-406.

37. Stergiou, N., Harbourne, R., Cavanaugh, J. Optimal movement variability: a new theoretical perspective for neurologic physical therapy. J Neurol Phys Ther, 2006. 30(3): p. 120-9.

38. Hamill, J., et al. A dynamical systems approach to lower extremity running injuries. Clin Biomech (Bristol, Avon), 1999. 14(5): p. 297-308.

39. Sanders T.L., et al. Incidence of anterior cruciate ligament tears and reconstruction: a 21-year population-based study. Am J Sports Med, 2016. 44(6): p. $1502-7$.

40. Kaeding, C.C., Léger-St-Jean B., Magnussen, R.A. Epidemiology and diagnosis of anterior cruciate ligament injuries. Clin Sports Med, 2017. 36(1): p. 1-8.

41. Paterno MV, et al. Incidence of contralateral and ipsilateral anterior cruciate ligament (ACL) injury after primary ACL reconstruction and return to sport. Clin J Sport Med, 2012. 22(2): p. 116-21.

42. Hewett TE, et al. The 2012 ABJS Nicolas Andry Award: The sequence of prevention: a systematic approach to prevent anterior cruciate ligament injury. Clin Orthop Relat Res, 2012. 470(10): p. 2930-40.

43. Hajizadeh $\mathrm{M}$, et al. Knee kinematics and joint moments during stair negotiation in participants with anterior cruciate ligament deficiency and reconstruction: a systematic review and meta-analysis. PM R, 2016. 8(6): p. 563-579.

44. Herzog W, Read LJ. Lines of action and moment arms of the major forcecarrying structures crossing the human knee joint. J Anat, 1993. 182(Pt 2): p. 213-230. 
45. Georgoulis A.D., et al. Three-dimensional kinematics of the tibiofemoral joint in ACL-deficient and reconstructed patients shows increased tibial rotation. Oper Tech Orthop, 2005. 15(1): p. 49-56.

46. Davis K. Quantifying joint coordination variability in anterior cruciate ligamentreconstructed individuals during walking [master's thesis]. Memphis: University of Tennessee Health Science Center, 2017.

47. Gribbin TC, et al. Differences in hip-knee joint coupling during gait after anterior cruciate ligament reconstruction. Clin Biomech (Bristol, Avon), 2016. 32: p. 64-71.

48. Pollard CD, et al. Altered lower extremity movement variability in female soccer players during side-step cutting after anterior cruciate ligament reconstruction. Am J Sports Med, 2015. 43(2): p. 460-5.

49. Hsu W-L, et al. Changes in interjoint coordination pattern in anterior cruciate ligament reconstructed knee during stair walking. JBSE, 2017. 12(2): p. 1600694.

50. Heiderscheit BC, Hamill J, van Emmerik RE. Variability of stride characteristics and joint coordination among individuals with unilateral patellofemoral pain. $\mathrm{J}$ Appl Biomech, 2002. 18(2): p. 110-21.

51. Chang R, van Emmerik RE, Hamill J. Quantifying rearfoot-forefoot coordination in human walking. J Biomech, 2008. 41(14): p. 3101-5.

52. Pollard CD, et al. Gender differences in lower extremity coupling variability during an unanticipated cutting maneuver. J Appl Biomech, 2005. 21(2): p. 14352.

53. Blache, Y., et al. Asymmetry of inter-joint coordination during single leg jump after anterior cruciate ligament reconstruction. Int J Sports Med, 2017. 38(2): p. 159-67.

54. Moraiti, C.O., et al. Anterior cruciate ligament reconstruction results in alterations in gait variability. Gait Posture, 2010. 32(2): p. 169-75.

55. Shabani, B., et al. Gait knee kinematics after ACL reconstruction: $3 D$ assessment. Int Orthop, 2015. 39(6): p. 1187-93.

56. Tashman, S., et al. Abnormal rotational knee motion during running after anterior cruciate ligament reconstruction. Am J Sports Med, 2004. 32(4): p. 97583.

57. Dhillion, M.S., Bali, K., Prabhakar, S. Proprioception in anterior cruciate ligament deficient knees and its relevance in anterior cruciate ligament reconstruction. Indian J Orthop, 2011. 45(4): p. 294-300.

58. Wang, H., et al. Correlation among proprioception, muscle strength, and balance. J Phys Ther Sci, 2016. 28(12): p. 3468-72.

59. Siegel, L., Vandenakker-Albanese, C., Siegel, D. Anterior cruciate ligament injuries: anatomy, physiology, biomechanics, and management. Clin J Sport Med, 2012. 22(4): p. 349-55. 
60. Fox, A.S. Change-of-direction biomechanics: is what's best for anterior cruciate ligament injury prevention also best for performance? Sports Med, 2018. Epub.

61. Kobayashi, H., et al. Mechanisms of the anterior cruciate ligament injury in sports activities: a twenty-year clinical research of 1,700 athletes. J Sports Sci Med, 2010. 9(4): p. 669-75. 


\section{APPENDIX A. SAMPLE MATLAB SCRIPT USED TO CALCULATE THE MEAN PHASE MAGNITUDE AND JOINT COORDINATION VARIABILITY}

The following script was used to make the aforementioned calculations in this study. It is configured to analyze two subject types given the joint angles over 101 normalized time points. The function must be given the couplings that are to be investigated and a cell number for the purpose of organization in an Excel spreadsheet to facilitate calling the function for a large number of couplings. Sub-phase transition frame numbers must be predetermined if the analyzed movement is to be partitioned. 


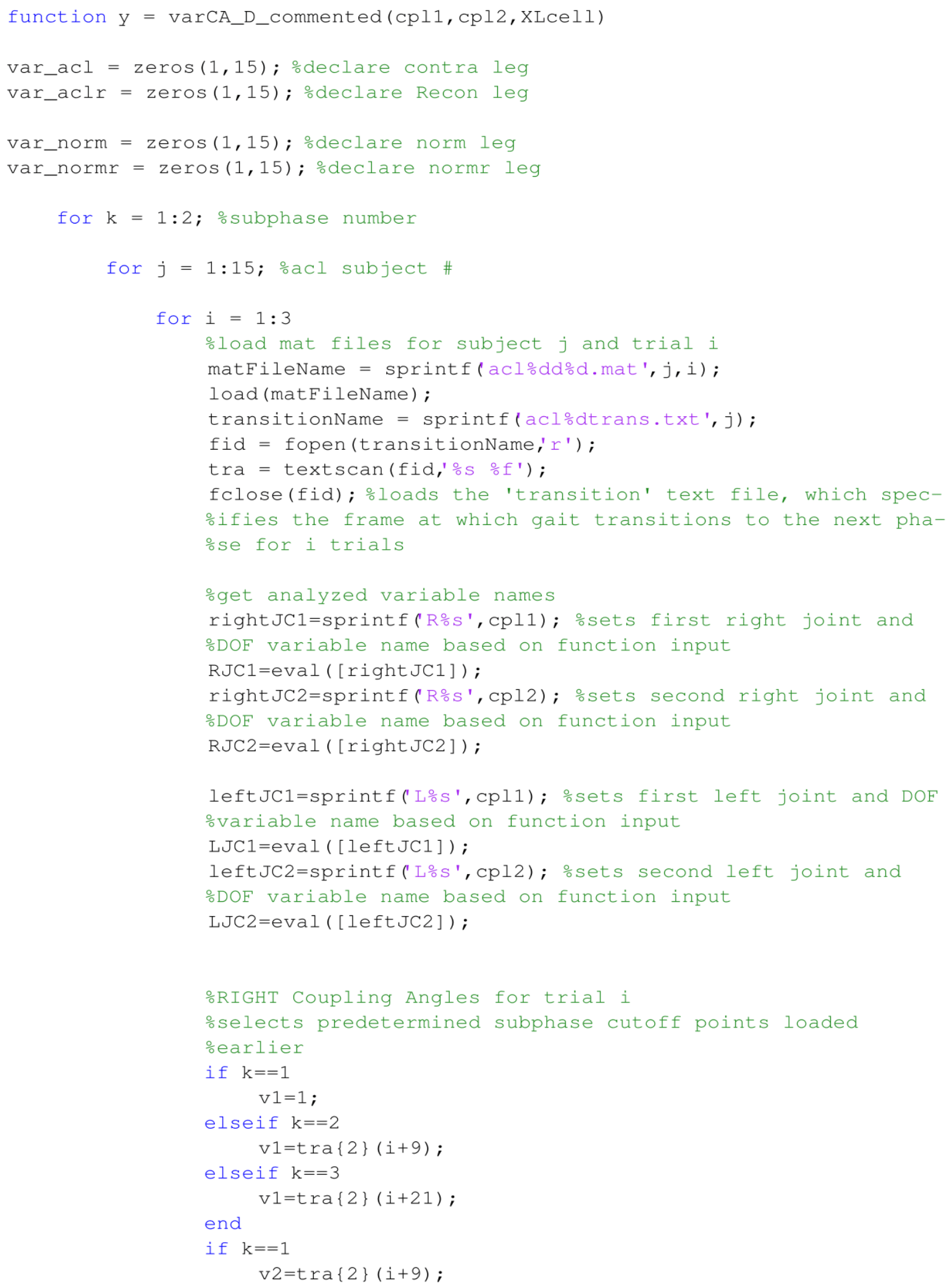




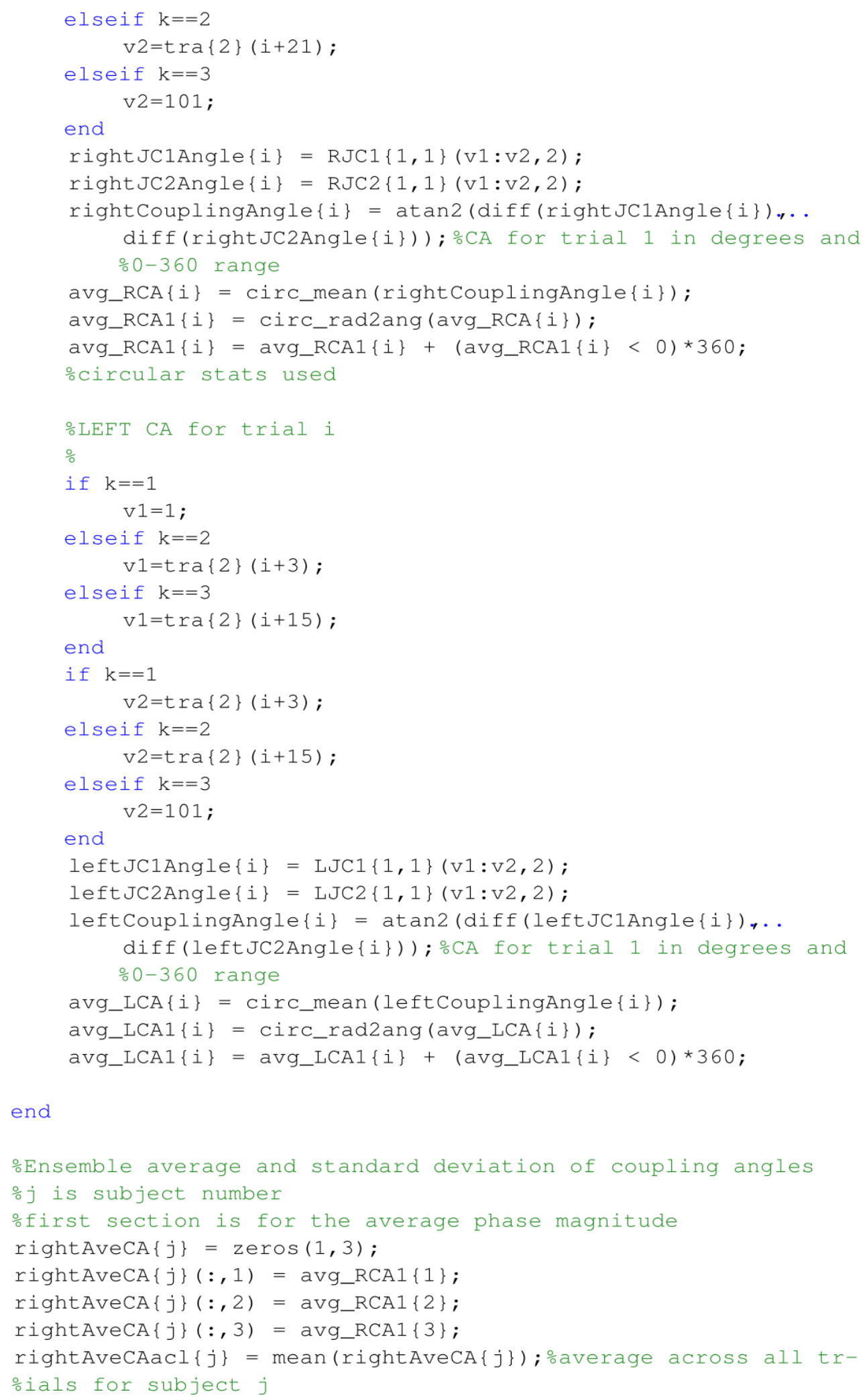




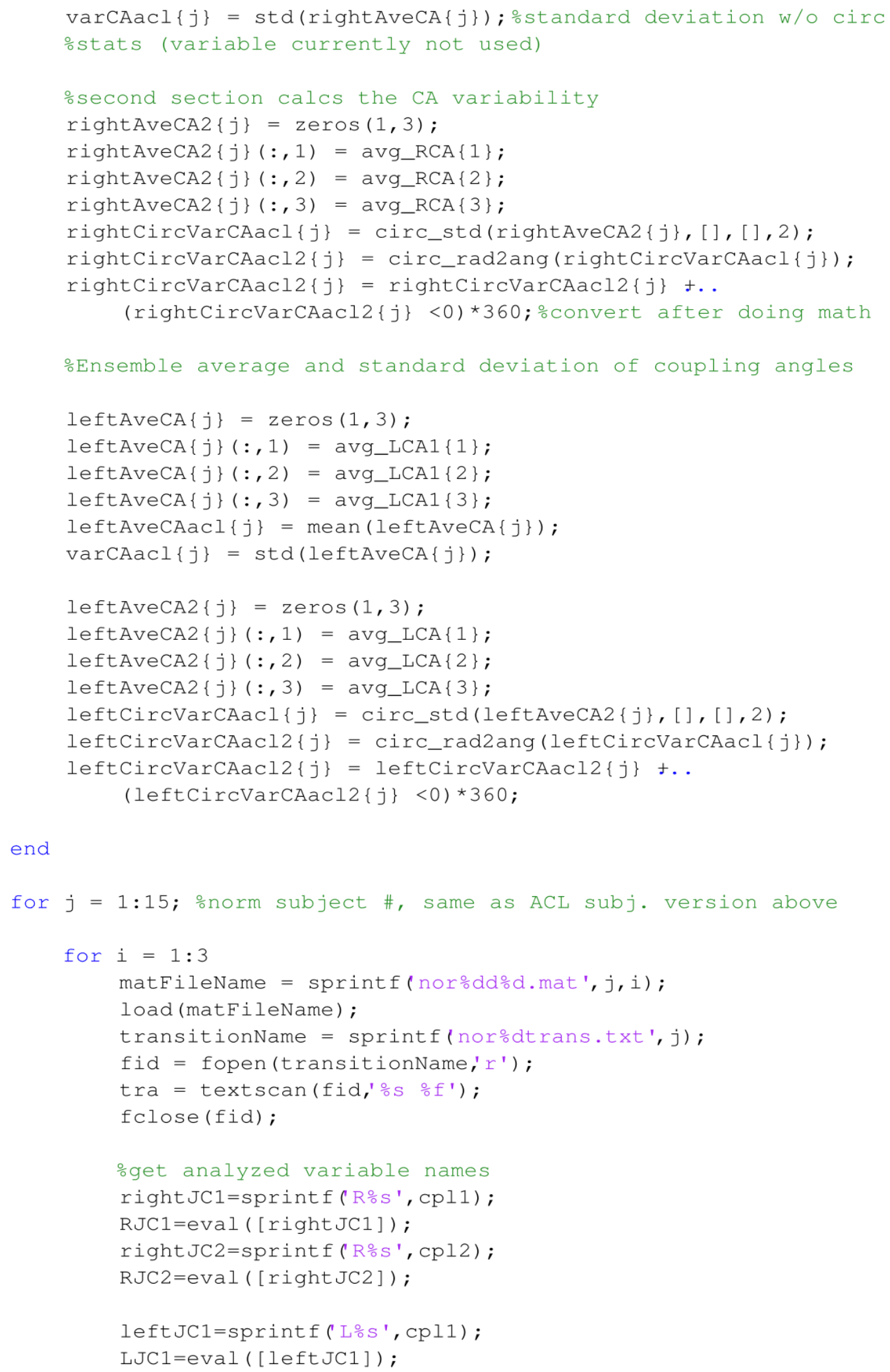




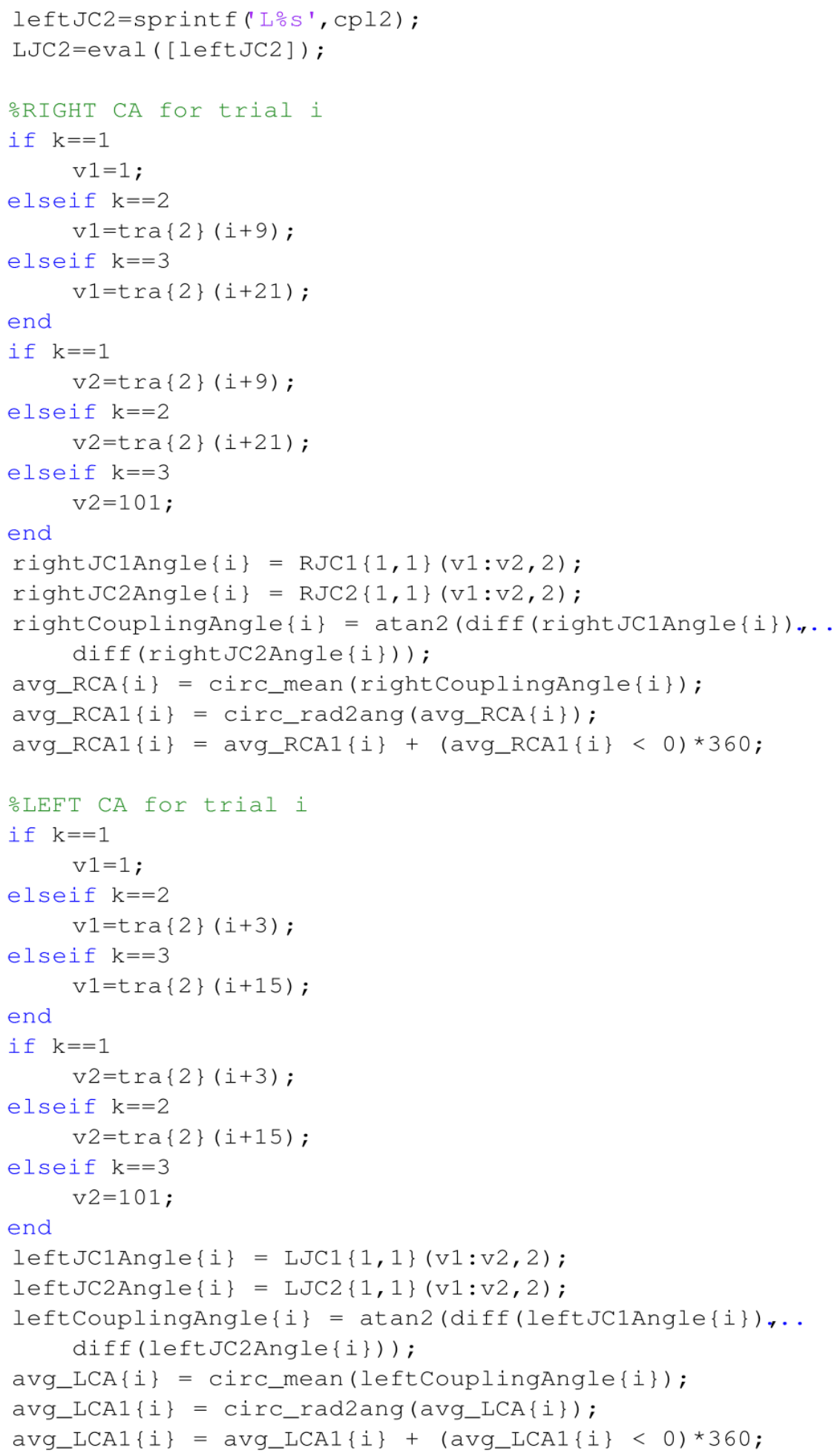




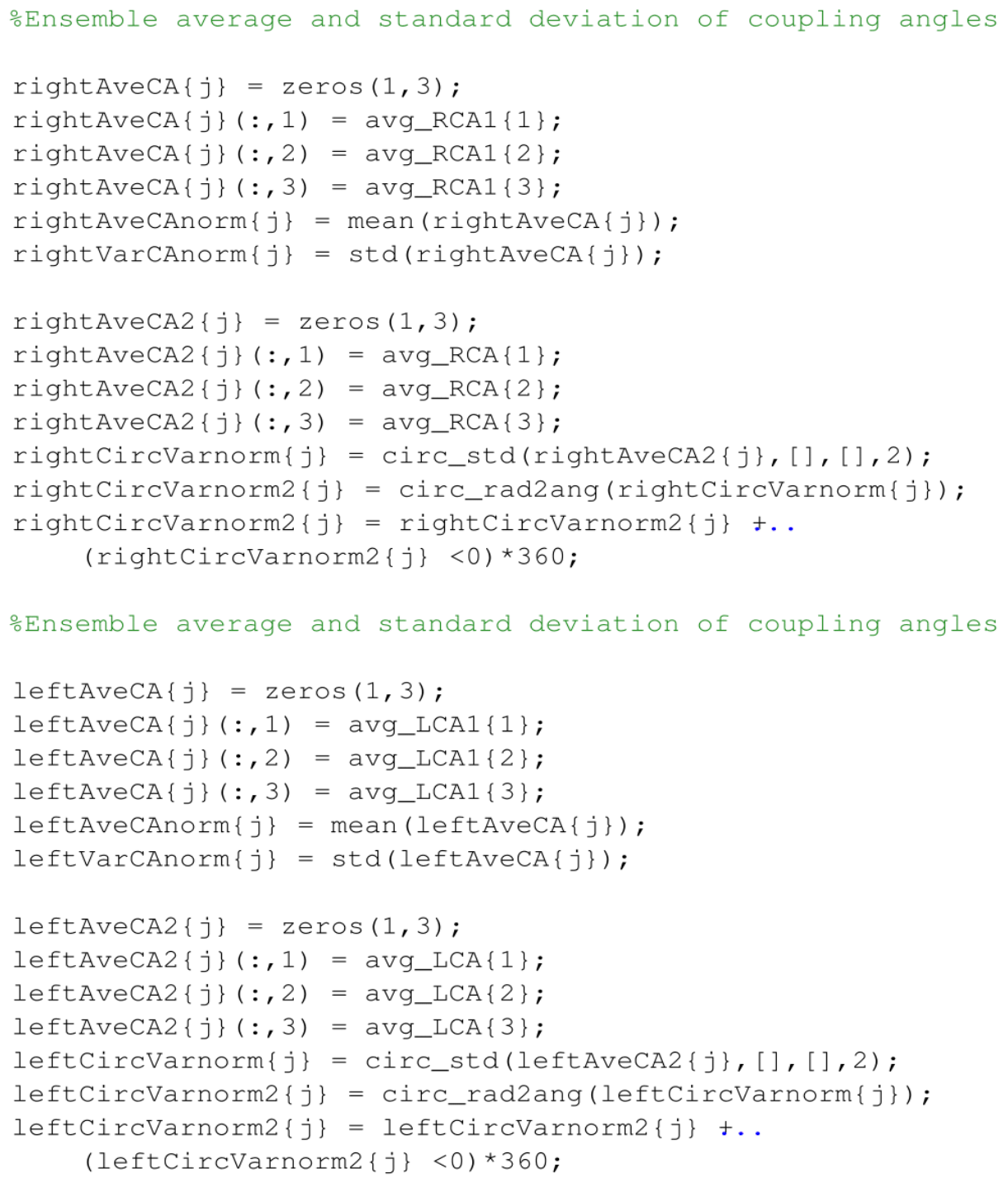




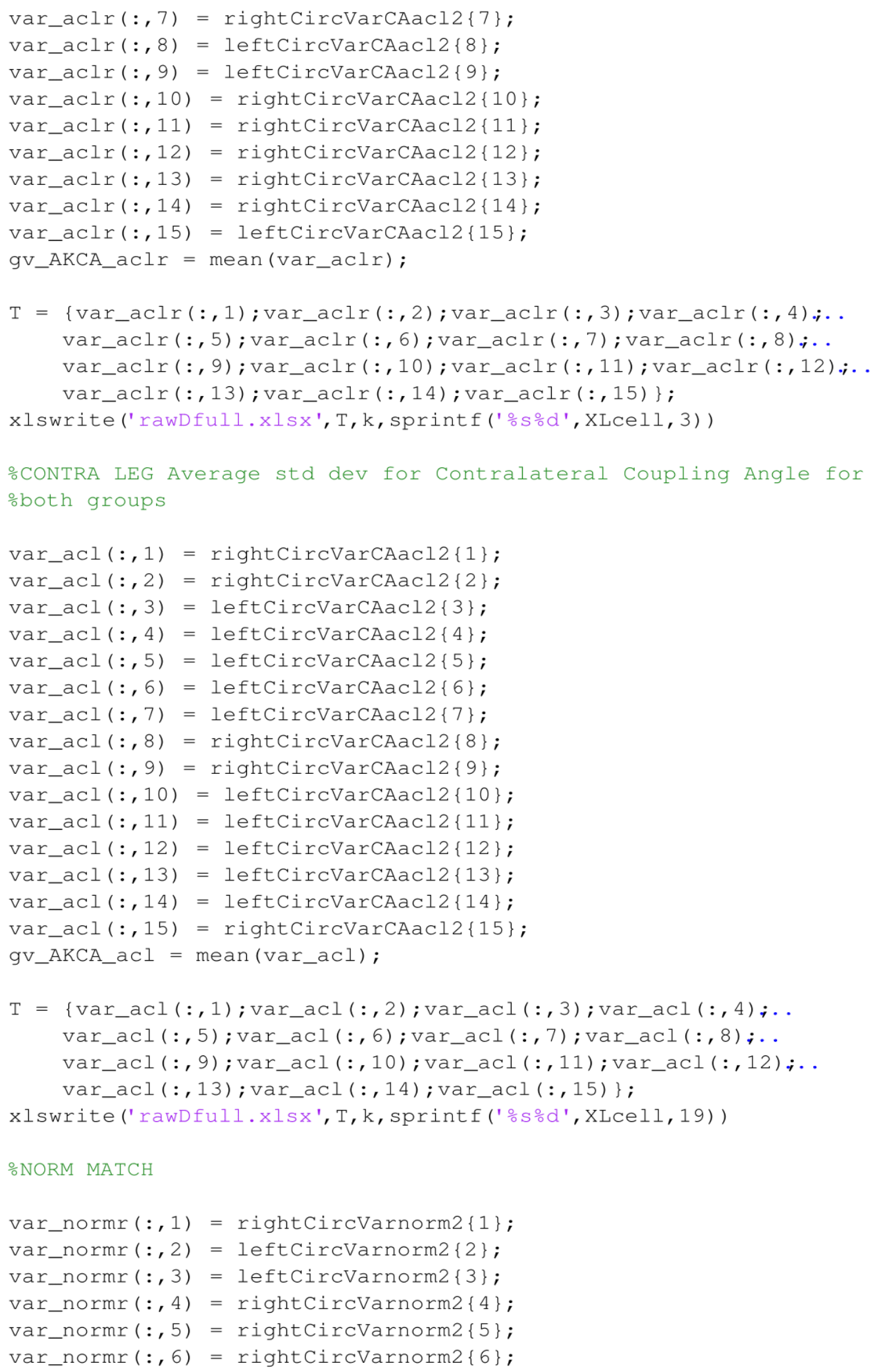




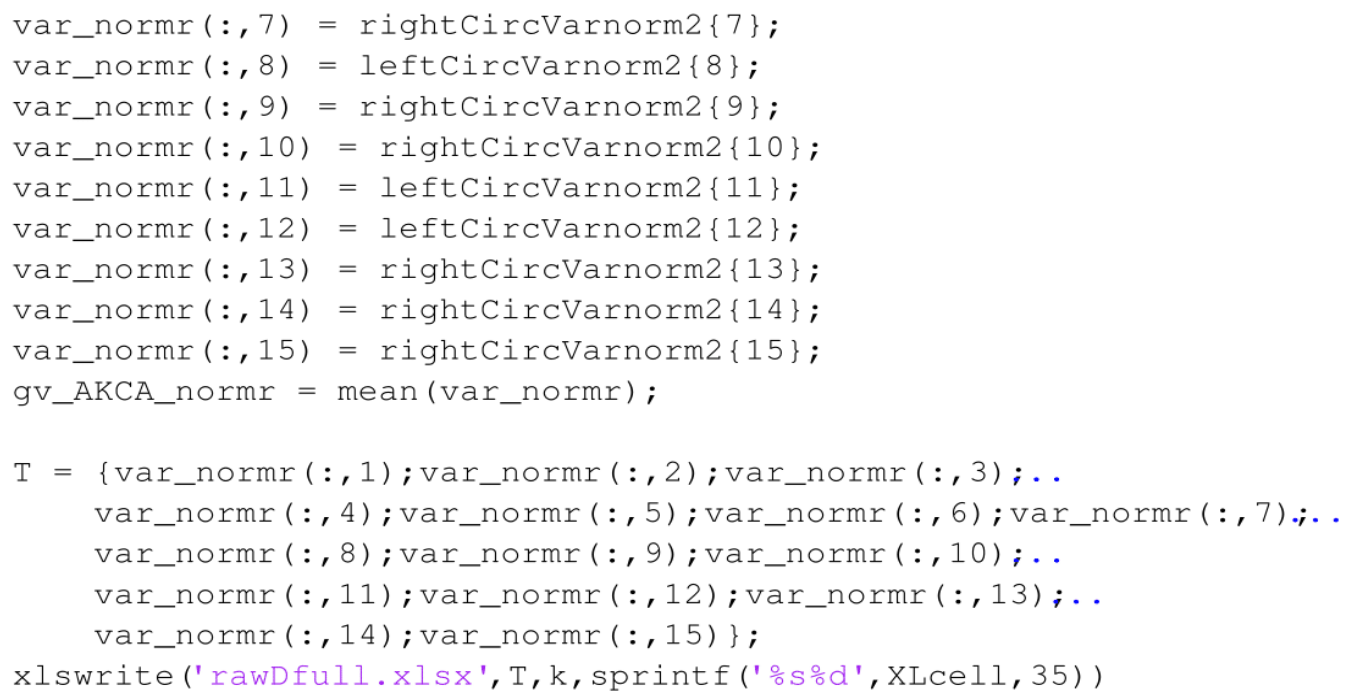




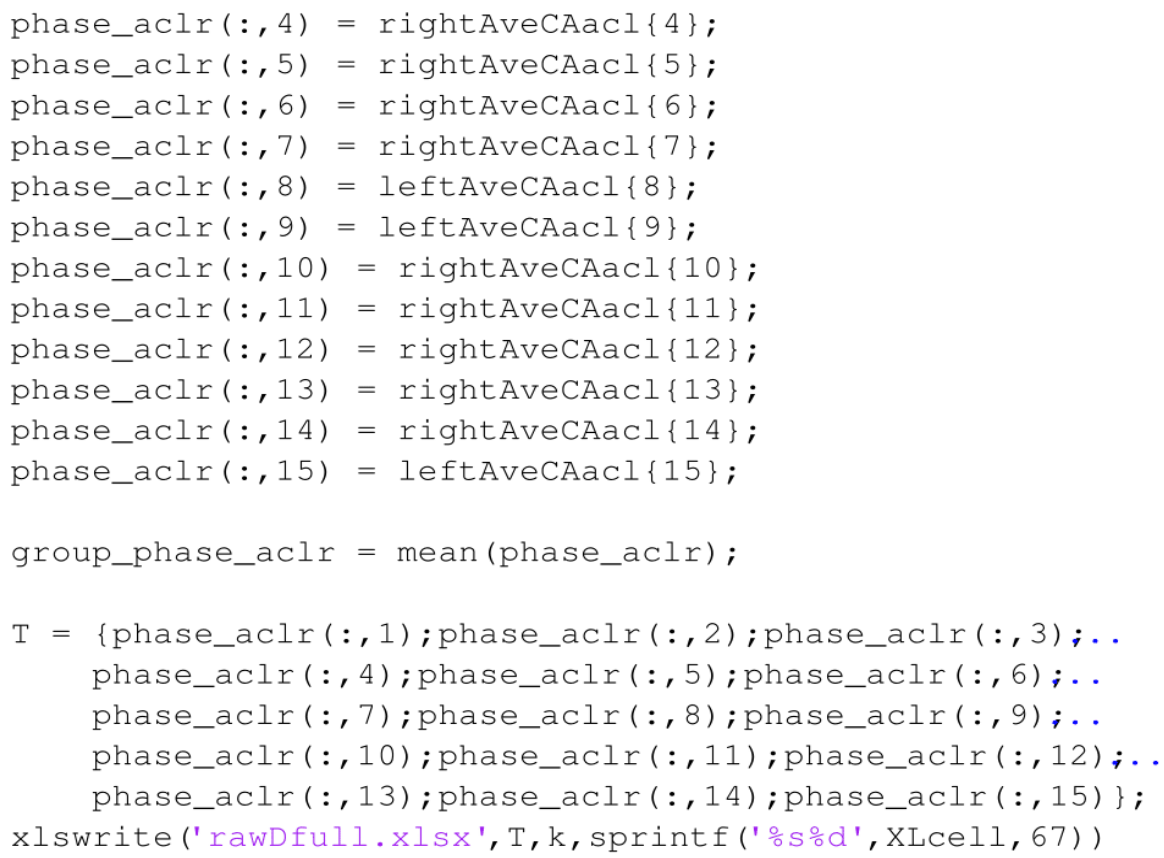

end 


\section{APPENDIX B. COMPLETE SET OF JOINT COORDINATION VARIABILITY ENSEMBLE AVERAGES FOR STAIR ASCENT AND DESCENT}

Table B-1. Joint coordination variability during stair ascent: mean (SD)

\begin{tabular}{|c|c|c|c|c|c|}
\hline $\begin{array}{c}\text { Stance } \\
\text { Sub-Phase }\end{array}$ & Coupling & $\operatorname{ACLR}\left(^{\circ}\right)$ & $\operatorname{ACLC}\left({ }^{\circ}\right)$ & Normal $\left(^{\circ}\right)$ & NormalC $\left(^{\circ}\right)$ \\
\hline PU & $\mathrm{KF}-\mathrm{AF}$ & $5.1(3.3)$ & $3.3(3.1)$ & $6.9(4.1)$ & $4.6(2.1)$ \\
\hline PU & KF-HF & $3.3(2.9)$ & $3.4(2.3)$ & $5.7(3.3)$ & $2.4(2.1)$ \\
\hline PU & KF-HR & $3.5(2.2)$ & $2.9(1.6)$ & $3.8(1.9)$ & $3.0(2.0)$ \\
\hline PU & KF-HA & $2.9(2.4)$ & $2.5(2.0)$ & $3.2(2.1)$ & $3.3(2.6)$ \\
\hline PU & KF-KR & $4.3(3.1)$ & $4.3(3.3)$ & $5.6(3.1)$ & $4.5(2.9)$ \\
\hline PU & KF-KA & $2.7(1.9)$ & $2.8(1.7)$ & $3.4(1.4)$ & $3.0(1.9)$ \\
\hline PU & KR-AF & $21.4(16.7)$ & $23.8(19.9)$ & $32.1(14.4)$ & $21.4(12.5)$ \\
\hline PU & KR-HF & $3.8(3.1)$ & $3.2(2.8)$ & $4.5(2.0)$ & $3.8(2.1)$ \\
\hline PU & KR-HR & $18.4(15.1)$ & $18.9(13.5)$ & $31.9(21.7)$ & $24.4(20.1)$ \\
\hline PU & KR-HA & $11.7(9.3)$ & $12.4(7.7)$ & $17.5(9.4)$ & $15.4(9.5)$ \\
\hline PU & KR-KA & $17.6(17.7)$ & $16.9(14.6)$ & $25.7(17.0)$ & $22.6(17.7)$ \\
\hline PU & KA-AF & $15.9(17.0)$ & $14.2(15.1)$ & $20.6(17.7)$ & $13.6(8.8)$ \\
\hline PU & KA-HF & $2.0(1.9)$ & $2.5(1.6)$ & $2.0(1.3)$ & $2.5(1.5)$ \\
\hline PU & KA-HR & $17.5(16.2)$ & $19.8(13.4)$ & $18.0(14.4)$ & $15.5(16.6)$ \\
\hline PU & KA-HA & $7.6(6.4)$ & $9.4(5.9)$ & $7.7(7.3)$ & 7.5 (3.8) \\
\hline PU & AF-HF & $4.5(3.1)$ & $3.3(2.8)$ & $6.7(4.1)$ & $4.8(1.7)$ \\
\hline PU & AF-HR & $21.0(15.3)$ & $19.8(17.7)$ & $27.7(19.8)$ & $20.8(14.5)$ \\
\hline PU & AF-HA & $16.7(12.5)$ & $10.9(6.4)$ & $25.1(17.8)$ & $15.7(13.2)$ \\
\hline PU & HF-HR & $2.9(1.6)$ & $2.7(1.5)$ & $3.1(1.8)$ & $2.9(1.5)$ \\
\hline PU & HF-HA & $2.5(2.4)$ & $2.1(1.3)$ & $2.8(2.0)$ & $2.5(1.6)$ \\
\hline PU & HR-HA & $11.3(3.6)$ & $12.3(8.1)$ & $12.3(10.6)$ & $12.1(8.7)$ \\
\hline $\mathrm{FC}$ & $\mathrm{KF}-\mathrm{AF}$ & $14.8(9.6)$ & $16.0(17.3)$ & $14.2(12.2)$ & $9.5(7.1)$ \\
\hline $\mathrm{FC}$ & KF-HF & $17.3(13.3)$ & $10.5(4.7)$ & $13.8(10.3)$ & $17.2(15.9)$ \\
\hline $\mathrm{FC}$ & KF-HR & $37.6(25.4)$ & $26.7(17.4)$ & $29.7(19.1)$ & $30.5(21.3)$ \\
\hline $\mathrm{FC}$ & KF-HA & $25.3(16.0)$ & $16.9(11.7)$ & $18.3(18.3)$ & $14.8(13.1)$ \\
\hline $\mathrm{FC}$ & KF-KR & $32.8(22.2)$ & $22.9(19.0)$ & $25.6(22.0)$ & $15.9(11.8)$ \\
\hline $\mathrm{FC}$ & KF-KA & $39.1(22.4)$ & $26.0(17.2)$ & $31.5(25.0)$ & $23.9(22.2)$ \\
\hline $\mathrm{FC}$ & $\mathrm{KR}-\mathrm{AF}$ & $12.8(9.3)$ & $20.1(20.4)$ & $14.8(12.4)$ & $12.2(8.1)$ \\
\hline $\mathrm{FC}$ & KR-HF & $8.9(5.0)$ & $8.7(4.9)$ & $12.1(12.0)$ & $7.0(3.7)$ \\
\hline $\mathrm{FC}$ & KR-HR & $25.7(19.5)$ & $22.2(16.5)$ & $22.3(16.0)$ & $24.0(16.7)$ \\
\hline $\mathrm{FC}$ & KR-HA & $14.3(9.6)$ & $12.2(7.6)$ & $14.2(14.6)$ & $11.1(8.2)$ \\
\hline $\mathrm{FC}$ & KR-KA & $27.1(16.2)$ & $22.6(11.8)$ & $24.5(20.6)$ & $19.7(19.8)$ \\
\hline $\mathrm{FC}$ & KA-AF & $12.9(9.8)$ & $18.4(15.2)$ & $15.4(19.1)$ & $8.7(5.2)$ \\
\hline $\mathrm{FC}$ & KA-HF & $13.6(13.4)$ & $11.9(13.5)$ & $10.4(6.0)$ & $12.3(14.7)$ \\
\hline $\mathrm{FC}$ & KA-HR & 37.7 (21.6) & $26.9(22.3)$ & $29.4(21.4)$ & $33.8(20.8)$ \\
\hline $\mathrm{FC}$ & KA-HA & $13.5(12.8)$ & $11.2(12.3)$ & $16.6(17.2)$ & $10.3(10.3)$ \\
\hline $\mathrm{FC}$ & AF-HF & $7.4(3.3)$ & $8.7(5.2)$ & $8.8(5.2)$ & $8.6(6.2)$ \\
\hline
\end{tabular}


Table B-1. (Continued)

\begin{tabular}{llcccc}
\hline $\begin{array}{c}\text { Stance } \\
\text { Sub-Phase }\end{array}$ & Coupling & ACLR $\left({ }^{\circ}\right)$ & ACLC $\left({ }^{\circ}\right)$ & Normal $\left({ }^{\circ}\right)$ & NormalC $\left({ }^{\circ}\right)$ \\
\hline FC & AF-HR & $12.8(11.6)$ & $18.7(18.0)$ & $10.9(6.4)$ & $8.8(7.7)$ \\
FC & AF-HA & $10.7(9.2)$ & $12.8(11.5)$ & $9.1(7.3)$ & $9.7(6.3)$ \\
FC & HF-HR & $17.7(17.5)$ & $8.9(5.9)$ & $13.9(9.3)$ & $20.0(19.1)$ \\
FC & HF-HA & $7.1(3.8)$ & $6.5(3.8)$ & $11.5(14.5)$ & $9.2(5.6)$ \\
FC & HR-HA & $18.2(15.9)$ & $11.9(9.8)$ & $13.6(10.7)$ & $17.2(13.8)$ \\
\hline
\end{tabular}

Coupling names are presented as an amalgamation of two joint and motion combinations (i.e. KF-KA=knee flexion/extension-knee abduction/adduction). $\mathrm{K}=\mathrm{knee}, \mathrm{H}=\mathrm{hip}$, $\mathrm{F}=$ flexion/extension, $\mathrm{R}=$ rotation, $\mathrm{A}=$ abduction/adduction, $\mathrm{PU}=$ pull up, $\mathrm{FC}=$ forward continuance. 
Table B-2. Joint coordination variability during stair descent: mean (SD)

\begin{tabular}{|c|c|c|c|c|c|}
\hline $\begin{array}{c}\text { Stance } \\
\text { Sub-Phase }\end{array}$ & Coupling & $\operatorname{ACLR}\left(^{\circ}\right)$ & $\operatorname{ACLC}\left({ }^{\circ}\right)$ & Normal $\left(^{\circ}\right)$ & NormalC $\left(^{\circ}\right)$ \\
\hline FC & KF-AF & $6.4(5.6)$ & $6.6(3.8)$ & $5.5(3.0)$ & $7.1(6.2)$ \\
\hline $\mathrm{FC}$ & KF-HF & $5.7(3.0)$ & $7.8(4.2)$ & $8.9(4.4)$ & $7.7(4.2)$ \\
\hline $\mathrm{FC}$ & KF-HR & $16.1(9.4)$ & $12.2(9.7)$ & $11.4(6.0)$ & $11.3(6.5)$ \\
\hline $\mathrm{FC}$ & KF-HA & $8.8(4.5)$ & $8.0(4.8)$ & $10.2(5.3)$ & $7.2(4.5)$ \\
\hline $\mathrm{FC}$ & KF-KR & $10.7(9.1)$ & $8.9(6.8)$ & $11.0(8.6)$ & $8.7(5.5)$ \\
\hline $\mathrm{FC}$ & KF-KA & $13.3(10.9)$ & $9.6(9.4)$ & $10.1(5.8)$ & $7.9(4.8)$ \\
\hline $\mathrm{FC}$ & $\mathrm{KR}-\mathrm{AF}$ & $8.9(8.4)$ & $6.1(2.7)$ & $5.4(3.0)$ & $7.1(4.3)$ \\
\hline $\mathrm{FC}$ & KR-HF & $13.6(6.4)$ & $11.3(5.4)$ & $13.2(11.7)$ & $16.0(13.5)$ \\
\hline $\mathrm{FC}$ & KR-HR & $20.2(10.7)$ & $16.2(12.2)$ & $17.2(15.1)$ & $15.4(9.4)$ \\
\hline $\mathrm{FC}$ & KR-HA & $11.7(7.4)$ & $13.5(10.4)$ & $15.2(12.3)$ & $10.8(5.0)$ \\
\hline $\mathrm{FC}$ & KR-KA & $20.0(13.7)$ & $15.4(10.9)$ & $17.8(18.5)$ & $13.3(8.2)$ \\
\hline $\mathrm{FC}$ & KA-AF & $6.0(7.0)$ & $5.1(4.3)$ & $4.5(4.5)$ & $7.1(7.9)$ \\
\hline $\mathrm{FC}$ & KA-HF & $18.3(22.8)$ & $14.1(7.8)$ & $20.5(16.6)$ & $19.2(12.8)$ \\
\hline $\mathrm{FC}$ & KA-HR & $28.0(22.3)$ & $36.6(24.8)$ & $26.6(14.6)$ & $35.2(21.8)$ \\
\hline $\mathrm{FC}$ & KA-HA & $15.7(13.9)$ & $15.1(15.3)$ & $16.6(14.2)$ & $12.9(10.3)$ \\
\hline $\mathrm{FC}$ & AF-HF & $5.4(4.0)$ & $4.3(2.6)$ & $6.2(4.7)$ & $5.9(7.2)$ \\
\hline $\mathrm{FC}$ & AF-HR & $8.5(4.0)$ & $5.6(3.8)$ & $5.9(3.6)$ & $6.7(4.9)$ \\
\hline $\mathrm{FC}$ & AF-HA & $7.3(7.0)$ & $4.2(3.3)$ & $4.7(3.2)$ & $6.6(7.9)$ \\
\hline $\mathrm{FC}$ & HF-HR & $17.2(21.0)$ & $18.3(16.2)$ & $22.6(19.8)$ & $24.3(16.4)$ \\
\hline $\mathrm{FC}$ & HF-HA & $10.0(10.3)$ & $9.9(6.5)$ & $12.8(9.0)$ & $13.1(12.2)$ \\
\hline $\mathrm{FC}$ & HR-HA & $16.7(13.4)$ & $16.9(9.6)$ & $18.4(11.6)$ & $21.5(12.9)$ \\
\hline $\mathrm{CL}$ & $\mathrm{KF}-\mathrm{AF}$ & $4.9(2.7)$ & $4.8(1.6)$ & $4.1(2.4)$ & $4.9(2.9)$ \\
\hline $\mathrm{CL}$ & KF-HF & $3.8(2.5)$ & $3.7(2.8)$ & $2.5(1.5)$ & $3.8(2.0)$ \\
\hline $\mathrm{CL}$ & KF-HR & $4.7(2.5)$ & $3.5(1.8)$ & $3.8(1.9)$ & $3.6(2.1)$ \\
\hline CL & KF-HA & $3.9(2.5)$ & $3.1(2.1)$ & $3.9(2.3)$ & $3.1(2.1)$ \\
\hline $\mathrm{CL}$ & KF-KR & $4.8(2.9)$ & $3.2(1.7)$ & $3.9(2.1)$ & $3.8(1.7)$ \\
\hline $\mathrm{CL}$ & KF-KA & $3.6(1.8)$ & $2.5(1.8)$ & $2.5(1.3)$ & $3.0(1.7)$ \\
\hline CL & $\mathrm{KR}-\mathrm{AF}$ & $17.7(14.0)$ & $18.5(11.1)$ & $18.0(17.5)$ & $23.7(18.3)$ \\
\hline CL & KR-HF & $12.2(11.4)$ & $10.1(4.7)$ & $17.1(13.9)$ & $13.7(11.3)$ \\
\hline CL & KR-HR & $17.5(14.4)$ & $18.6(16.9)$ & $34.9(20.0)$ & $16.7(10.6)$ \\
\hline CL & KR-HA & $21.6(12.9)$ & $31.1(18.8)$ & $29.2(22.3)$ & $28.3(22.4)$ \\
\hline $\mathrm{CL}$ & KR-KA & $14.5(8.3)$ & $22.5(14.9)$ & $24.6(15.3)$ & $15.7(15.2)$ \\
\hline $\mathrm{CL}$ & KA-AF & $15.3(17.0)$ & $16.2(12.5)$ & $14.6(11.2)$ & $18.0(15.4)$ \\
\hline CL & KA-HF & $10.1(9.3)$ & $10.3(8.3)$ & $12.9(7.3)$ & $15.1(16.4)$ \\
\hline CL & KA-HR & $15.7(13.6)$ & $15.2(12.3)$ & $29.1(20.5)$ & $15.7(14.5)$ \\
\hline CL & KA-HA & $14.7(14.7)$ & $21.6(17.6)$ & $23.2(12.4)$ & $18.9(17.2)$ \\
\hline $\mathrm{CL}$ & AF-HF & $10.3(7.9)$ & $11.9(6.0)$ & $15.3(15.5)$ & $18.3(19.7)$ \\
\hline CL & AF-HR & $12.9(16.1)$ & $12.9(7.2)$ & $24.9(20.1)$ & $15.2(9.1)$ \\
\hline $\mathrm{CL}$ & AF-HA & $11.4(10.3)$ & $14.6(10.4)$ & $20.8(15.3)$ & $20.5(21.1)$ \\
\hline $\mathrm{CL}$ & HF-HR & $11.2(5.2)$ & $10.0(7.9)$ & $14.6(5.6)$ & $13.6(7.7)$ \\
\hline $\mathrm{CL}$ & HF-HA & $11.8(6.5)$ & $11.8(9.4)$ & $15.1(9.9)$ & $14.1(14.7)$ \\
\hline $\mathrm{CL}$ & HR-HA & $19.5(17.0)$ & $20.0(16.0)$ & $32.8(14.5)$ & $27.6(21.2)$ \\
\hline
\end{tabular}




\section{Table B-2. (Continued)}

Coupling names are presented as an amalgamation of two joint and motion combinations (i.e. $\mathrm{KF}-\mathrm{KA}=\mathrm{knee}$ flexion/extension-knee abduction/adduction). $\mathrm{K}=\mathrm{knee}, \mathrm{H}=\mathrm{hip}$, $\mathrm{F}=$ flexion/extension, $\mathrm{R}=$ rotation, $\mathrm{A}=$ abduction/adduction, $\mathrm{FC}=$ forward continuance, $\mathrm{CL}=$ controlled lowering. 


\section{VITA}

Bryan R. Simrak was born in Pasadena, CA in 1986 and grew up in the San Gabriel Valley of Los Angeles. He studied Physics at the University of California Santa Barbara from 2005 to 2009 where he earned his Bachelor's degree. He worked as a Manufacturing and Field Service Engineer until 2016 when he opted to pursue graduate studies. Bryan was admitted to the joint Biomedical Engineering program to study biomechanics at the University of Memphis and University of Tennessee Health Science Center and graduated with a Master's degree in 2018. 